\title{
Transcriptome Analysis of Acer truncatum Seeds Reveals Candidate Genes Related to Oil Biosynthesis and Fatty Acid Metabolism
}

\author{
Qiuyue Ma, Shushun Li, Jing Wen, Lu Zhu, Kunyuan Yan, and Qianzhong Li \\ Institute of Leisure Agriculture, Jiangsu Academy of Agricultural Sciences Nanjing, 210014, China \\ Shuxian Li and Bin Zhang \\ Southern Modern Forestry Collaborative Innovation Center, Nanjing Forestry University, Nanjing, \\ 210037, China
}

\begin{abstract}
ADDITIONAL INDEX wORDs. differentially expressed gene, unsaturated fatty acids, RNA sequencing, transcription factors
ABstract. Acer truncatum seeds are an excellent source of beneficial natural compounds, including high levels of unsaturated fatty acids (UFAs), that promote health. Recently, $A$. truncatum has emerged as an oil crop. Therefore, the transcriptomes of $A$. truncatum seeds at 70, 85, 100, 115, 145, 180 days after flowering (DAF) were analyzed to gain a better understanding of the transcriptional and translational regulation of seed development and oil biosynthesis. A total of 28,438 genes were identified, and 3069/2636, 3288/3438, 1319/2750, and 5724/5815 upregulated/downregulated genes were identified when comparing different samples with 85 DAF seeds. Sixteen lipid metabolism pathways with 754 differentially expressed genes (DEGs) were identified, including 34 DEGs associated with UFA biosynthesis. A phylogenetic analysis revealed that six putative fatty acid desaturase $(F A D)$ genes clustered into five $F A D$ groups. A quantitative real-time polymerase chain reaction analysis indicated that the temporal expression patterns of oil biosynthesis genes and transcription factors were largely similar to the RNA sequencing results. The results of this study will enhance the current understanding of oil metabolism in $A$. truncatum seeds and allow new methods of improving oil quality and seed yield in the future.
\end{abstract}

Acer truncatum is a versatile oil-producing woody tree. It is well-known for its colorful leaves and high value as an ornamental, medicinal, and oil plant (Ma et al., 2005, 2020; Yang et al., 2017). In 2011, the seed oil was approved as a New Resource Food by the National Health and Family Planning Commission of the People's Republic of China, with potentially important implications for the fields of food and medicine. Previous studies of $A$. truncatum have mainly focused on its seed oil (Chen et al., 2017; Liang et al., 2019; Wang et al., 2018) that contains UFAs, including oleic acid (C18:1), linoleic acid (C18:2), $\alpha$-linolenic acid (C18:3), and nervonic acid (C24:1) (Liu et al., 2003; Wang et al., 2006). In particular, the proportions of $\mathrm{C} 18: 1$ and $\mathrm{C} 18: 2$ are higher than those in other edible oils, including peanut (Arachis hypogaea), rapeseed (Brassica napus), and sunflower (Helianthus annuus) oils (Zhao et al., 2006). In addition, $A$. truncatum seeds are rich in phytosterols, polysaccharides, and flavonoids (Hu et al., 2017; Liang et al., 2019). Because of the potential value of $A$. truncatum seeds as a source of "healthy" oil, it is important to understand seed development and oil biosynthesis in $A$. truncatum to identify strategies for increasing the oil content and quality through breeding and management practices.

Received for publication 29 Oct. 2020. Accepted for publication 20 Nov. 2020 Published online 30 December 2020.

This work was funded by the Natural Science Foundation of China (32001357), the Independent Innovation Fund Project of Agricultural Science and Technology in Jiangsu Province (CX(20)3155), and the Technology Innovation and Extension Project of Forestry Science in Jiangsu Province (LYKJ[2018]14). We also thank Robbie Lewis, MSc, from Liwen Bianji, Edanz Group China (www.liwenbianji.cn/ac), for editing a draft of this manuscript.

Q.L. is the corresponding author. E-mail: qianzhongli@jaas.ac.cn.

This is an open access article distributed under the CC BY-NC-ND license (https://creativecommons.org/licenses/by-nc-nd/4.0/).
Although some researchers have reported the seed oil and UFA contents, there is limited information regarding the regulatory mechanisms controlling seed development and oil biosynthesis. RNA sequencing (RNA-seq) is a powerful tool used to study global gene expression and identify genes that are functionally important under different physiological conditions or developmental stages (Alotaibi et al., 2020; Wang et al., 2019). Recently, an RNA-seq analysis has been used to examine the regulatory mechanisms associated with seed development, fatty acid (FA) biosynthesis, and UFA accumulation in some woody oilseed crops, such as tree peony [Paeonia section Moutan (Liang et al., 2019)], camelina [Camelina sativa (Mudalkar et al., 2014)], olive [Olea europaea (Muñoz-Mérida et al., 2013)], and physic nut [Jatropha curcas (Grover et al., 2014)]. However, such research of $A$. truncatum is limited. The first comprehensive study characterizing the gene expression in A. truncatum seeds was conducted by Wang et al. (2018). They compared the transcriptomes of two genotypes of $A$. truncatum seeds and identified candidate genes that influenced the verylong-chain fatty acid (VLCFA) accumulation. They identified 10 (differentially expressed genes) DEGs that were potentially involved in VLCFA synthesis. However, they only studied two genotypes of $A$. truncatum seeds. No studies have performed a transcriptome analysis of developing $A$. truncatum seeds with a focus on the biosynthesis of FAs and triacylglycerol (TAG). Oil accumulates in $A$. truncatum seeds as the seed develops, which takes $\approx 6$ months from pollination to seed maturity. In addition, seed morphology and the levels of chemical compounds vary greatly at different developmental stages, and the expressions of genes related to FA metabolism vary (Wang et al., 2019). The temporal and spatial characteristics of the transcriptome, how FA and TAG biosynthesis pathways are regulated, and 
how these pathways affect the oil content, composition, and accumulation during seed development are unclear. Therefore, it is necessary to explore the transcriptome of developing seeds to gain a better understanding of the regulation of lipid metabolism.

In this study, the FA content and composition in developing seeds were analyzed and a time-series analysis of transcriptomic data was performed to reveal DEGs in six phases of $A$. truncatum seed development. This study aimed to assess the transcriptional profiles and identify the main functional genes related to FA and TAG biosynthesis at five time points. Our systematic identification of the core biological processes involved in oil synthesis and the newly identified transcription factors (TFs) will help us to further explore the molecular regulatory mechanisms of oil biosynthesis and metabolism in developing $A$. truncatum seeds.

\section{Materials Methods}

Plant materials. $A$. truncatum plants were grown in the Jiangsu Province Aceraceae Germplasm Repository (Lishui District, Nanjing, China, lat. $31^{\circ} 65^{\prime} \mathrm{N}$, long. $119^{\circ} 02^{\prime} \mathrm{E}$ ) under natural conditions. Seeds were collected at six time points during their development (70, 85, 100, 115, 145, and 180 DAF) from three individuals of "Lishui" provenance. Seeds collected from the same individuals were used for RNA sequencing and oil content measurements. The samples were frozen in liquid nitrogen immediately following collection and stored at $-80^{\circ} \mathrm{C}$ until further use.

LIPID ANALYSIS. Fifty seeds from each developmental stage were weighed and dried at $65{ }^{\circ} \mathrm{C}$ for $72 \mathrm{~h}$. The seed oil was extracted with petroleum ether $\left(30\right.$ to $\left.60^{\circ} \mathrm{C}\right)$, and the distillation temperature was kept at $65^{\circ} \mathrm{C}$ for $24 \mathrm{~h}$. Following Gao (2006), $0.2 \mathrm{~mL}$ oil was dissolved in $2 \mathrm{~mL}$ benzene/petroleum ether $(1: 1$, $\mathrm{v} / \mathrm{v})$ and then mixed with $2 \mathrm{~mL} \mathrm{KOH}-\mathrm{CH}_{3} \mathrm{OH}\left(0.4 \mathrm{~mol} \cdot \mathrm{L}^{-1}\right)$ to generate FA methyl esters. The resulting FAs were analyzed using the gas chromatography-mass spectrometry (GC-MS) method as described by Jiao et al. (2015). The amounts of FAs were calculated by combining the peak areas of the FA methyl esters according to standards (Zhang et al., 2018).

RNA EXTRACTION, LIBRARY CONSTRUCTION, AND SEQUENCING. Total RNA was extracted from developing seeds at 70, 85, 100, 115,145 , and 180 DAF from three plants (three biological repeats) using TRIzol Reagent (Thermo Fisher Scientific, Waltham, MA) according to the manufacturer's instructions. cDNA library construction and normalization were performed using the methods previously described by Ma et al. (2020). The 18 cDNA libraries were sequenced on an Illumina HiSeq X10 (Illumina, San Diego, CA) and 150-bp paired-end reads were generated. The paired-end reads that remained after quality control (low-quality reads containing $>10 \%$ unknown bases or $50 \%$ low-quality bases) were mapped to our $A$. truncatum reference genome (Ma et al., 2020) using HISTA2 version 2.0.4 (Kim et al., 2015).

DifFerential EXPRESSiOn ANALYSIS AND FUNCTIONAL ANALYSIS. The DEGs was performed with the Deseq 2 package (Wang et al., 2010) using a model based on the negative binomial distribution $[P<0.05$ ( $P$ was adjusted using Benjamini and Hochberg's approach)] with the 85 DAF sample serving as the control. All DEGs were mapped to gene ontology (GO) terms (Ashburner et al., 2000) to conduct the GO enrichment analysis. The hypergeometric test (Mao et al.,
2015) was used to identify significantly enriched GO terms, and the probability was adjusted by Bonferroni correction $(P<$ 0.05) (Kirk, 2006). The Kyoto Encyclopedia of Genes and Genomes (KEGG) pathway-related database was used to perform the DEG pathway enrichment analysis (Kanehisa et al., 2008).

Phylogenetic analysis of $\boldsymbol{A}$. Truncatum fatty desaturase GENES. The expression patterns of six annotated $A$. truncatum fatty desaturase $(F A D)$ genes were displayed as a heat map according to $\log 10$ [fragments per kilobase per million (FPKM) +1 ] values. A phylogenetic analysis was performed using the maximum likelihood method with MEGA 5.2 (Tamura et al., 2011). Bootstrap tests were performed with 1000 replicates for statistical reliability. FAD genes of Arabidopsis thaliana, Sesamum indicum, Glycine max, and O. europaea were downloaded from NCBI.

Quantitative REAL-TIME POLYMERASE CHAin REACTION ANALYSIS OF GENES INVOLVED IN OIL BIOSYNTHESIS. Quantitative real-time polymerase chain reaction (qRT-PCR) was performed to validate the results of the RNA-seq analysis using 16 differentially expressed genes and five TFs involved in FA biosynthesis and metabolism. Specific primers were designed with Primer5 (Supplemental Table 1). Total RNAs were extracted as described previously. The first-strand cDNA was reverse-transcribed from $1 \mu \mathrm{g}$ total RNA with $\mathrm{M}-\mathrm{MLV}$ reverse transcriptase (Promega, Madison, WI). The qRT-PCR was performed using SYBR Premix ExTaq ${ }^{\mathrm{TM}}$ (TaKaRa Bio, Kusatsu, Japan) on a Step One Plus Real-Time PCR System (Applied Biosystems, Thermo Fisher Scientific). There were three biological replicates for each time point from three different trees (nested with three technical replicates). The relative gene expression levels were normalized to the internal control Actin (Wang et al., 2018) using the $2^{-\Delta \Delta \mathrm{T}}$ method (Livak and Schmittgen, 2001).

\section{Results}

MAJOR FA CONTENT AND COMPOSITION DIFFERENCES IN DEVELOPING SEEDS. To obtain a time series of oil content variation during seed development, A. truncatum seeds at 70, $85,100,115,145$, and 180 DAF were selected for oil content and composition measurements. The morphology of developing seeds at six different time points is shown in Fig. 1. The seeds maintained a low growth rate at the early stage (70-85 DAF), and the kernels rapidly swelled after 85 DAF. Five dominant components were observed in developing seeds, including palmitic acid (C16:0), stearic acid (C18:0), oleic acid (C18:1), linoleic acid (C18:2), and $\alpha$-linolenic acid (C18:3). As shown in Fig. 1, no UFAs were detected at 70 DAF. At 85 DAF, only C18:1 and C18:2 were detected, and the proportions were $0.46 \%$ and $0.63 \%$, respectively. The UFA proportions rapidly increased from 85 to 115 DAF. By 115 DAF, C16:0 and C18:0 had increased to $4.89 \%$ and $2.68 \%$, respectively, and $\mathrm{C} 18: 1, \mathrm{C} 18: 2$, and $\mathrm{C} 18: 3$ increased to $20.93 \%, 30.21 \%$, and $0.85 \%$, respectively. Therefore, the results showed that $85 \mathrm{DAF}$ was the key time point for FA synthesis. Overall, the content variation of the major components from 115 to $180 \mathrm{DAF}$ was not significant, thus providing a platform to elucidate how FAs are biosynthesized in $A$. truncatum seeds.

RNA SEQUENCING AND TRANSCRIPTOME ASSEMbLy. Eighteen libraries were sequenced from seeds collected at six stages of 


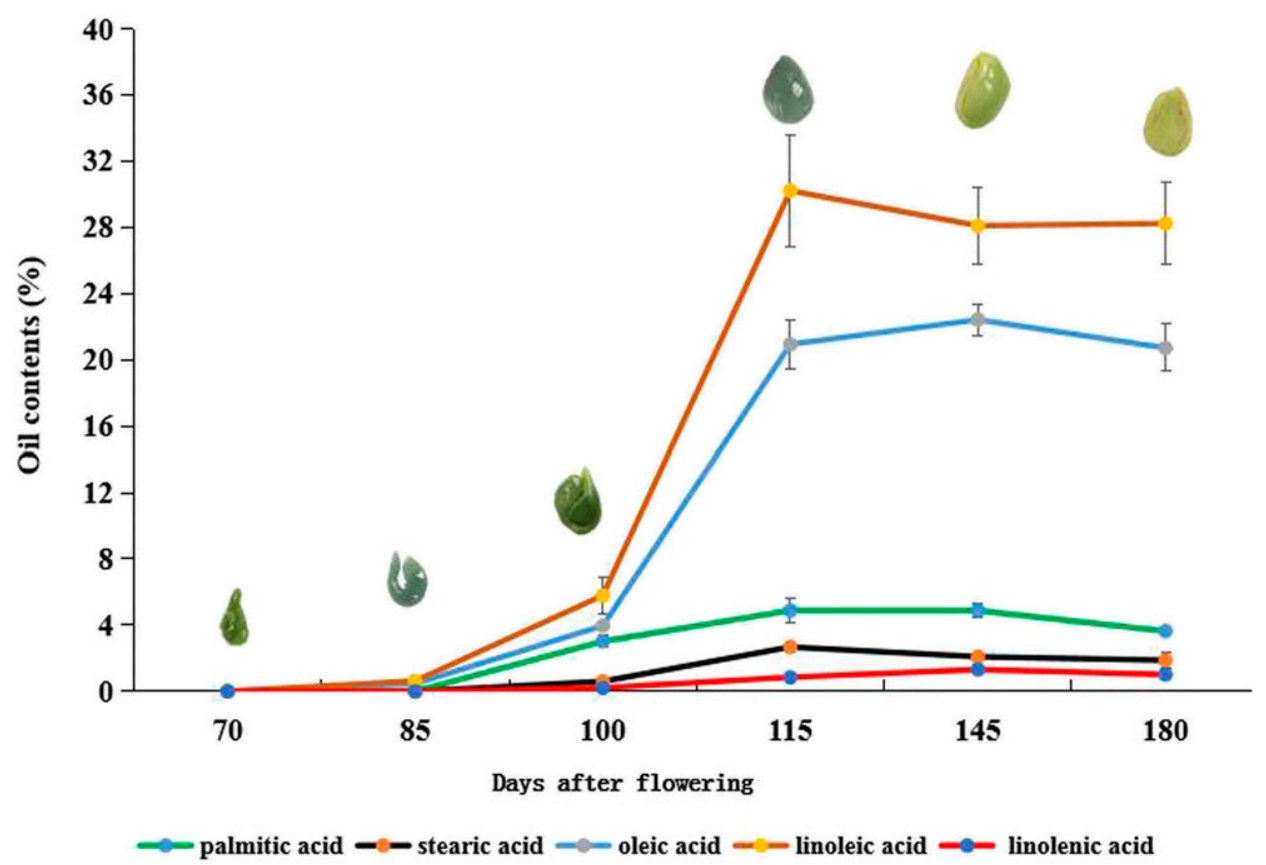

Fig. 1. Changes in fatty composition during Acer truncatum seed development. The x-axis represents days after flowering and the y-axis shows the percentage of different oil components.

development to provide a profile of the global transcriptome of A. truncatum seeds. A total of $129.56 \mathrm{~Gb}$ of data with an average of 49,335,115 raw sequencing reads and 47,987,471 clean reads per sample were obtained after the removal of poly$\mathrm{N}$ sequences and low-quality reads. The clean reads were submitted to the Sequence Read Archive (SRA) under study accession number SRS5367121 and biosample accession number SAMN12389479. The average ratio of clean reads to raw reads was $97.27 \%$. The clean reads were mapped to the $A$. truncatum genome we sequenced previously (Ma et al., 2020), with an average mapping percentage of $90.39 \%$ per sample. Overall, $73.71 \%$ to $84.08 \%$ of the reads were uniquely matched, whereas $6.80 \%$ to $12.83 \%$ of reads mapped to multiple positions (Supplemental Table 2). A total of 28,438 genes were identified.

DifFERENTIAL EXPRESSION ANALYSIS AND CLUSTERING OF DEGs. A differential expression analysis was conducted to characterize the temporal changes in global gene expression. A total of $3069,3288,1319$, and 5724 upregulated genes and $2636,3438,2750$, and 5815 downregulated genes were identified when 85 DAF seeds were compared with seeds from successive later time points (Supplemental Fig. 1A). The numbers of upregulated and downregulated genes were similar at different time points except for 145 vs. 85 DAF. The four comparisons with the 85 DAF sample were grouped into two main clusters based on the patterns of differential gene expressions: 85 to $100+100$ to 115 and 115 to $145+145$ to 180 DAF (Supplemental Fig. 1B). The clustered time points suggested that $A$. truncatum seeds were at similar developmental stages at the time points in those two clusters.

GENE ONTOLOGY FUNCTIONAL CLASSIFICATION AND ENRICHMENT ANALYSIS OF DEGs. GO functional annotation was performed for the DEGs identified during the four comparisons with the 85 DAF sample (Supplemental Fig. 2). The 33 functional groups in the biological process, cellular component, and molecular function categories were classified according to the GO annotations of the DEGs. The four comparisons exhibited similar patterns of GO functional classification; however, they differed in enrichment classifications (Supplemental Fig. 2). The "compound biosynthetic process" and "primary metabolic biosynthetic process" categories were enriched in the 100 vs. 85 DAF and 115 vs. 85 DAF comparisons, including the terms "nitrogen compound metabolic process," "regulation of cellular metabolic process,", "regulation of primary metabolism," and "RNA biosynthetic process," indicating that crude fat and various FAs were rapidly being synthesized (as shown in Fig. 1, the levels of various FAs peaked in the 115 DAF samples). "Cell part," "organelle," and "chromatin" were the main enriched terms in the cellular component category. Notably, in the 180 vs. 85 DAF sample (when desiccation occurs and the levels of FAs start to decrease), the "response to stress" and "degradation complex" (peptidase, hydrolase activity) terms were enriched.

KEGG FUNCTIONAL CLASSIFICATION AND PATHWAY ENRICHMENT ANALYSIS OF DEGs. To identify the major biological pathways active in developing $A$. truncatum seeds, the DEGs were mapped to the reference pathways in KEGG. The DEGs were assigned to $24 \mathrm{KEGG}$ terms under the six primary categories, including cellular processes, environmental information processing, genetic information processing, metabolism, and organismal systems (Supplemental Fig. 3). To elucidate the enriched biological functions during the four comparisons, an enrichment analysis of the 280 pathways to which the DEGs were mapped was performed (Supplemental Table 3). The results showed that ribosome processing and protein processing in the endoplasmic reticulum pathways were mainly enriched in the 100 DAF sample, when seeds were rapidly developing. Flavonoid biosynthesis and FA biosynthesis were also becoming significantly enriched during this period (Supplemental Fig. 4). The biosynthesis of secondary metabolites was significantly enriched after 115 DAF. Interestingly, in 115 vs. 85 DAF comparisons, 15 and 4 DEGs were significantly enriched in $\alpha$-linolenic acid and the linoleic acid metabolism pathway, respectively. This is consistent with fact that the UFA proportions rapidly increased from 85 to 115 DAF. In addition, the DEGs identified in the present study mapped to 16 of the 17 pathways for lipid metabolism (Supplemental Table 4). Specifically, 754 DEGs were associated with FA metabolism, 34 were associated with arachidonic acid metabolism, 34 were associated with UFA biosynthesis, 42 were associated with cutin, suberin, and wax biosynthesis, 44 were associated with FA biosynthesis, 42 were associated with FA degradation, 45 were associated with FA elongation, and 20 were associated with linoleic acid metabolism. Six FADs were identified and differentially expressed at different stages of seed 

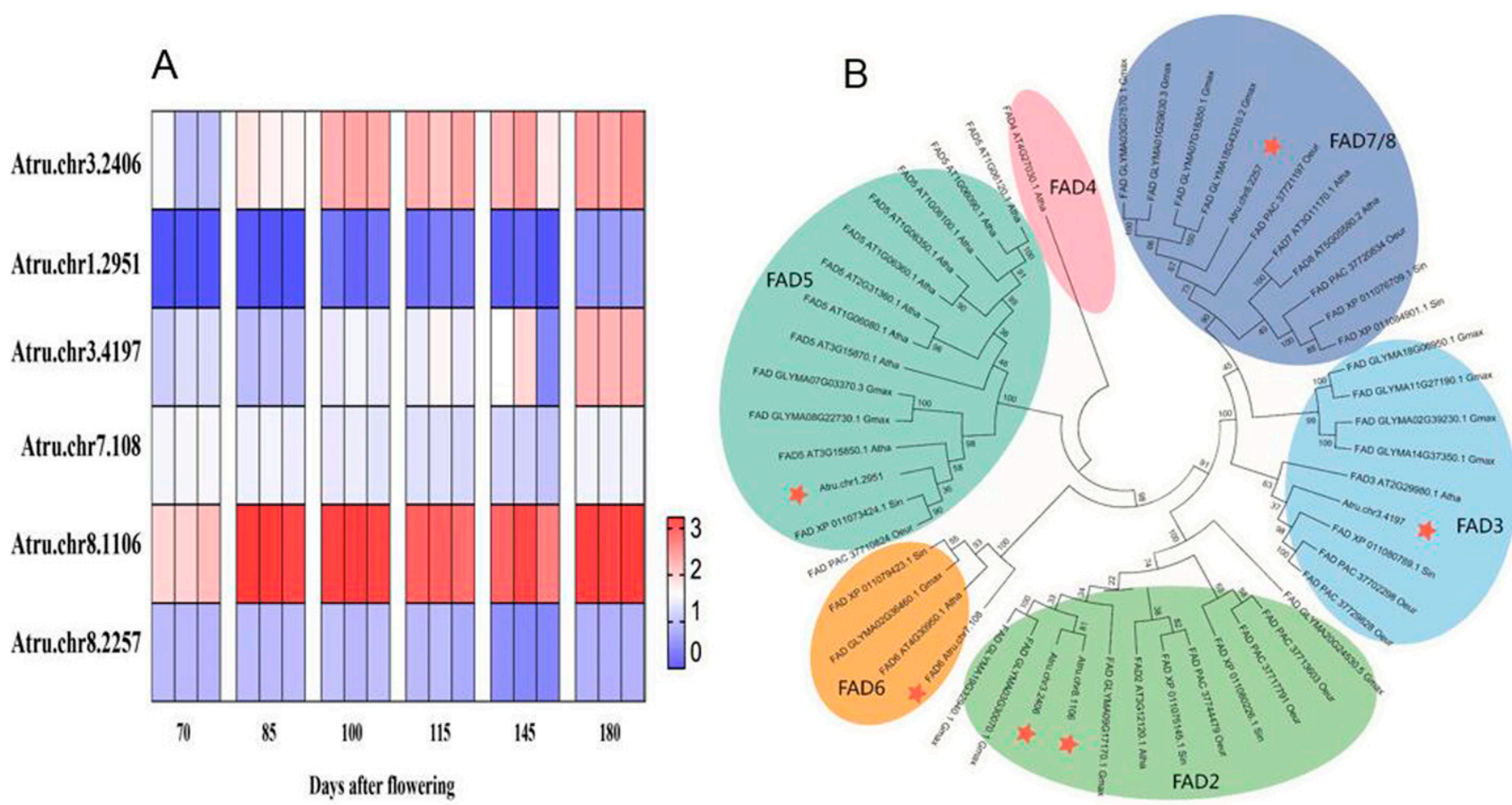

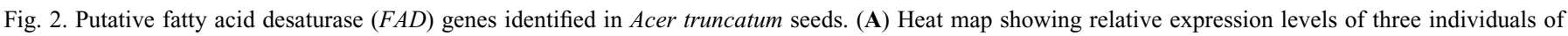
'Lishui' provenance at different seed development stages. (B) Phylogenetic tree depicting the relationships of Acer truncatum (Atru) FADs with other species: Arabidopsis thaliana (Atha), Sesamum indicum (Sin), Olea europaea (Oeur), and Glycine max (Gmax). The groups of FAD proteins are designated according to Dong et al. (2016). The heat map is displayed according to $\log _{10}$ [fragments per kilobase per million $($ FPKM $\left.)+1\right]$ values.

development (Fig. 2A), including two FAD2, one FAD3, one FAD5, one FAD6, and one FAD7/8 genes based on the phylogenetic analysis of $A$. thaliana. Interestingly, FAD4 was not found in $A$. truncatum, unlike in the other selected species (Fig. 2B). The DEGs related to lipid metabolism at different time points of $A$. truncatum seed development can provide important information about the key stages of oil accumulation and the mechanism regulating UFA synthesis.

QRT-PCR ANALYSIS OF OIL BIOSYNTHESIS GENES DURING THE FIVE TIME POINTS OF A. TRUNCATUM SEED DEVELOPMENT. TO validate the reliability of the RNA-seq results, 16 genes related to FA biosynthesis were selected for qRT-PCR validation (Fig. 3 ). The results indicated that ACC, FATA1, FATA2, and FATB were highly expressed at $85 \mathrm{DAF}$, and that $S A D 1, F A D 2$, and $F A D 5$ were highly expressed from 85 to 100 DAF. In addition, $P D A T$ and $D G A T$ are the key genes involved in triglyceride synthesis; we found that they were mainly highly expressed at 180 DAF. In general, the qRT-PCR results for oil biosynthesis genes, except $S A D 3$ and $F A D 6$, displayed a high degree of consistency with the RNA-seq results. These results confirmed the reliability of the RNA-seq data obtained during our study.

IDENTIFICATION AND EXPRESSION PROFILING OF FATTY ACID TAG BIOSYNTHESIS GENES. The 51 genes encoding key enzymes involved in FA biosynthesis were successfully identified based on the KEGG pathway analysis, and their expression levels were determined during different developmental stages (Fig. 4). The oil synthesis pathway in A. truncatum was constructed based on the results of the present and previous studies (Liao et al., 2018; Wang et al., 2019). In plants, the de novo synthesis of FAs occurs first in the plastid, with acetyl-CoA carboxylase (ACCase) catalyzing the biosynthesis of malonyl-CoA from
acetyl-CoA. ACCase catalyzes the rate-limiting step in FA biosynthesis. Its four subunits are biotin carboxylase $(\mathrm{BC})$, carboxyl transferase $\alpha(\mathrm{CT}-\alpha)$, carboxyl transferase $\beta$ (CT- $\beta)$, and biotin carboxyl carrier protein (BCCP). All subunits except $C T$ - $\beta$ were highly expressed during $A$. truncatum seed development. Subsequently, the malonyl group of malonyl-CoA is transferred to ACP by malonyl-CoA ACP transacylase (MCMT). Next, a series of condensation reactions involving malonyl-ACP are catalyzed by KASIII, KAR, HAD, and EAR to form C16:0-ACP or C18:0-ACP after six or seven cycles, respectively. Additionally, the $\mathrm{C} 16: 0-\mathrm{ACP}$ or $\mathrm{C} 18: 0-\mathrm{ACP}$ can be further catalyzed by $S A D$ to generate C16:1-ACP or C18:1ACP; three $S A D$ genes were identified and highly expressed, especially $S A D 1$ (Figs. 3 and 4). Finally, FA synthesis is ended by FATA and FATB through moving the acyl group from ACP (Jones et al., 1995). After this, LACS on the outer membrane of the plastid esterifies free FAs to generate an acyl-CoA pool (Rawsthorne, 2002; Wang et al., 2012). Some acyl-CoA enter the phosphatidylcholine pool, whereas other acyl-CoA are combined with glycerol-3-phosphate to form TAG. In the phosphatidylcholine pool, PC-C18:1 is converted to PC$\mathrm{C} 18: 2$ by FAD2, which is then converted to $\mathrm{PC}-\mathrm{C} 18: 3$ by FAD3. The C18:1 and C18:2 FAs together account for 52\% of the total FA content in A. truncatum. Previous studies have demonstrated that the accumulation of $\mathrm{C} 18: 2$ is correlated with the expression of FAD2/FAD6, whereas FAD3/FAD7/FAD8 expression contributes to the synthesis of C18:3 (Wang et al., 2019; Yin et al., 2018; Zhang et al., 2019). FAD2 and FAD3 exhibited higher expressions than the other FAD genes (Figs. 3 and 4), suggesting that they have an important role in UFA biosynthesis. 

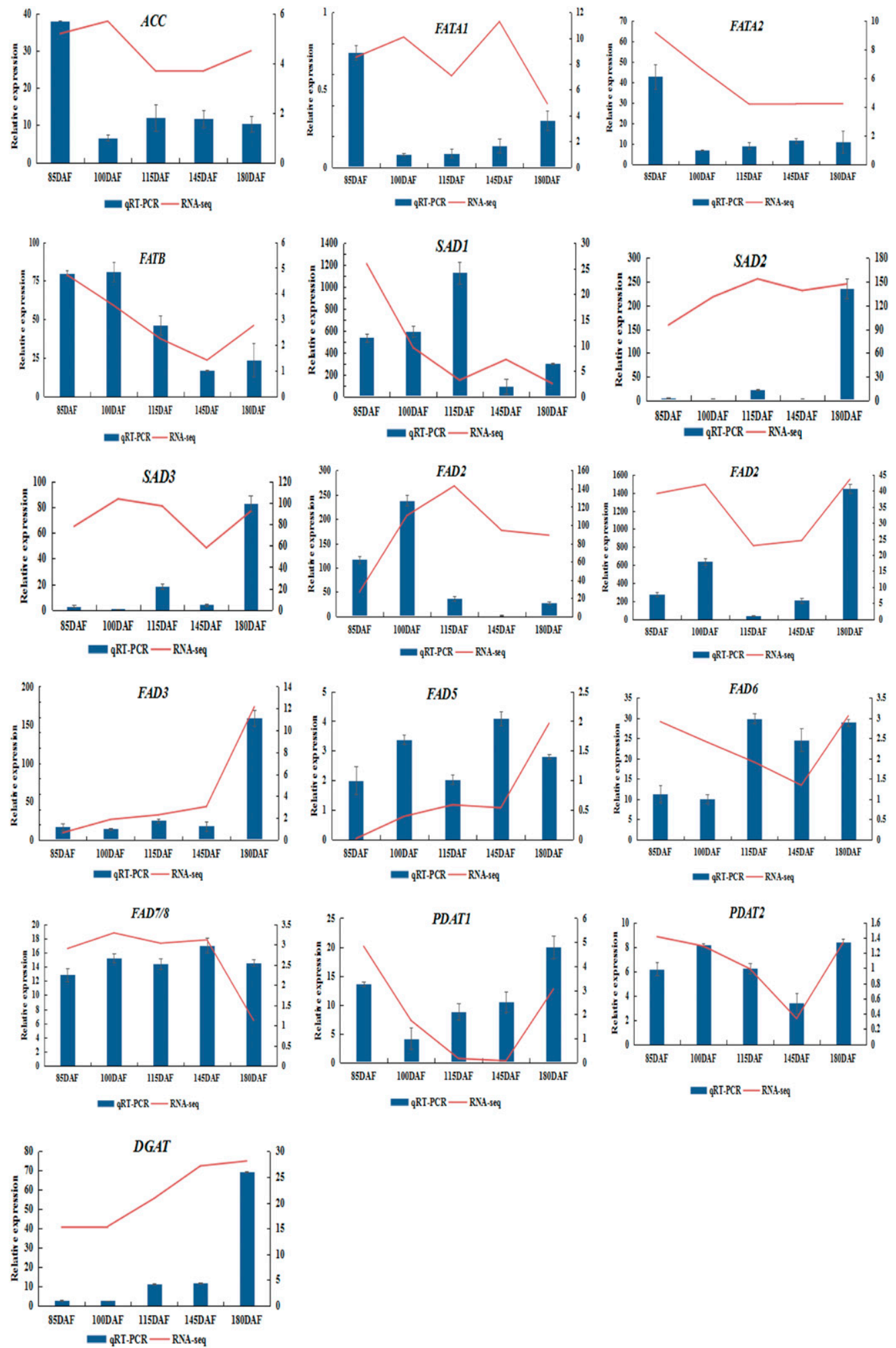

Fig. 3. Quantitative real-time polymerase chain reaction (qRT-PCR) validation of oil biosynthesis genes at five time points of Acer truncatum seed development. The relative expression levels of genes were normalized with the internal reference gene Actin (Wang et al., 2018). Three biological replicates (nested with three technical replicates) are represented by the error bars $(P<0.05)$.

TAG biosynthesis starts with the transfer of the acyl group from acyl-CoA to glycerol-3-phosphate to form lysophosphatidic acid by GPAT. In this study, nine GPAT genes were identified, but most of them were barely expressed; only two were relatively highly expressed during seed development. Subsequently, lysophosphatidic acid is dephosphorylated by LPAT and PAP to sn-1,2-diacylglycerol (DAG) (Dahlqvist et al., 2000; Lung and Weselake, 2006). The biosynthesis of 


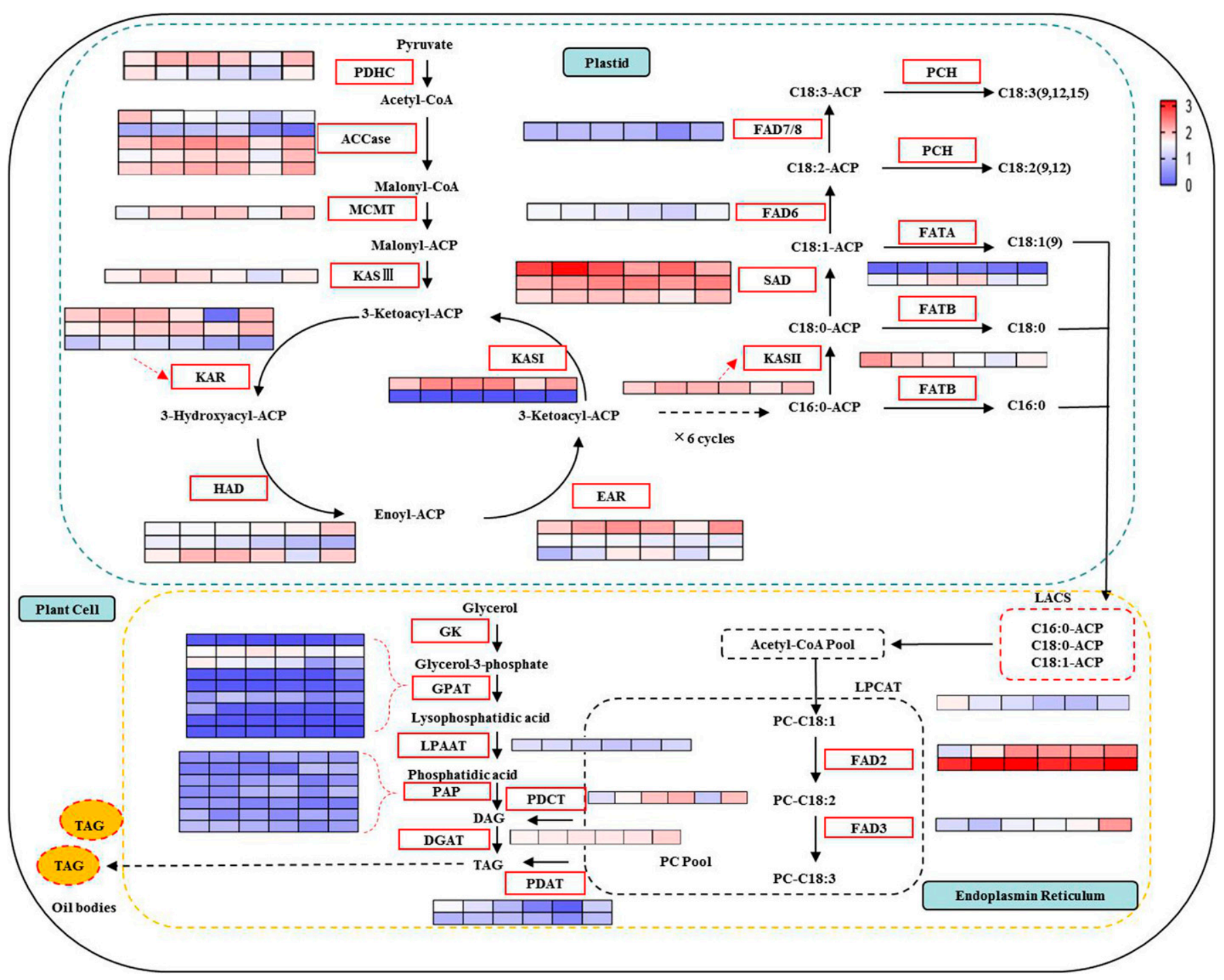

Fig. 4. The pathways of fatty acid biosynthesis. The icons next to the key enzymes represent the relative expression levels of the transcripts at $85,100,115,145$, and $180 \mathrm{~d}$ after flowering (DAF). The heat map is displayed according to $\log _{10}$ [fragments per kilobase per million $\left.(\mathrm{FPKM})+1\right]$ values. PDHC $=$ pyruvate dehydrogenase complex; ACCase = acetyl-CoA carboxylase; MCMT = malonyl-CoA-ACP transacylase; KASI/II/III = ketoacyl-ACP synthase I/II/III; KAR = ketoacyl-ACP reductase; $\mathrm{HAD}=$ hydroxyacyl-ACP dehydrase; $\mathrm{EAR}=$ enoyl-ACP reductase; $\mathrm{SAD}=$ stearoyl-ACP desaturase; FAD2/6 = oleate desaturase; FAD3/7/8 = linoleate desaturase; FATA/B = acyl-ACP thioesterase A/B; PCH = palmitoyl-CoA hydrolase LACS = long-chain acyl-CoA synthetase; LPCAT = lysophosphatidylcholine acyltransferase; GK = glycerol kinase; GPAT = glycerol-3-phosphate acyltransferase; LPAAT = 1-acylglycerol-3-phosphate acyltransferase; PAP = phosphatidic acid phosphatase; DGAT = diacylglycerol acyltransferase; PDAT = phospholipid:diacylglycerol acyltransferase.

TAG is ended by DGAT, which is a rate-limiting enzyme in the Kennedy pathway (Liao et al., 2018). The qRT-PCR results showed that DGAT had the highest expression at 180 DAF (Fig. $3)$. This indicated that TAG assembly and accumulation through the glycolysis pathway occurred in seeds at $\approx 180$ DAF.

IDENTIFICATION OF TRANSCRIPTION FACTORS INVOLVED IN OIL BIOSYNTHESIS AND DEPOSITION. TFs have important roles in transcription and can modulate gene expression as regulators (Riechmann et al., 2000). However, little is known about the transcriptional regulation of lipid biosynthesis in A. truncatum seeds. In this study, a total of 1049 TFs were identified during the seed oil accumulation stages using iTAK software (Zheng et al., 2016). The top six TF families in terms of sequence abundance were bHLH (59), HB (51), MYB-related (48), NAC (39), bZIP (32), and B3 (30) domain proteins. In previous studies, several TFs were shown to have important roles in seed oil biosynthesis, including wrinkled1 [WRI1 (Ji et al., 2018)], leafy cotyledon1 [LECl (Mu et al., 2008; Shen et al., 2010)], fusca3 [FUS3 (Zhang et al., 2016)], and Abscisic acid insensitive3 [ABI3 (Rohde et al., 2010)]. They were also identified among our transcripts, including one WRII, two LECl, one FUS3, and one $A B I 3$. To further determine the expression differences of the TFs, these five TFs were selected for qRTPCR analysis during the five seed development stages (Fig. 5). The results showed that their expression tendencies were consistent with the RNA-seq results. However, the expressions of the two homologous $L E C l$ genes differed greatly. The expression of $L E C l$ was significantly higher than that of LEC1.1. In short, the expression trends of WRI1 and LEC1.1 increased and those of FUS3 and $A B I 3$ decreased. This might have been due to tissue differences or specific regulatory differences in the expression patterns. Further studies will be 

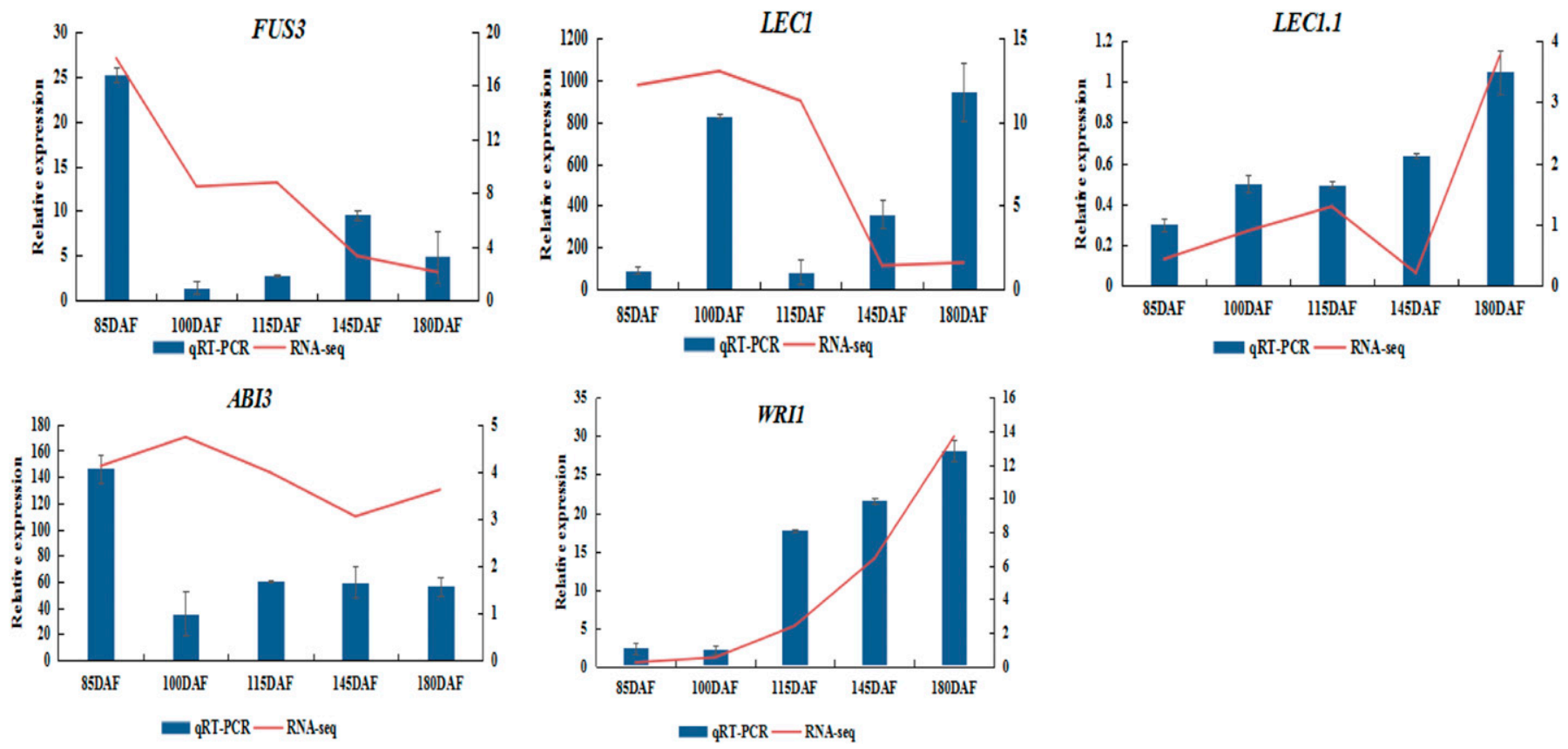

Fig. 5. Quantitative real-time polymerase chain reaction (qRT-PCR) validation of fusca3 (FUS3), leafy cotyledon1 ( $L E C 1)$, leafy cotyledon1 ( $L E C 1.1)$, Abscisic acid insensitive3 (ABI3), and wrinkled1 (WRII) at five time points of Acer truncatum seed development. The relative expression levels of genes were normalized with the internal reference gene Actin (Wang et al., 2018). Three biological replicates (nested with three technical replicates) are represented by the error bars.

needed to elucidate the regulatory mechanisms of the TFs involved in oil accumulation in A. truncatum seeds. In summary, our results provide some valuable clues to help understand the molecular mechanisms of FA and lipid biosynthesis.

\section{Discussion}

Dynamic Changes in Gene EXPRESSion DURING SEED DEVELOPMENT. A. truncatum is an important woody tree species that produces seeds with high levels of UFAs (Ma et al., 2020). Studying the key stages of seed development and the molecular mechanism of oil synthesis is a critical step in improving the quantity and quality of $A$. truncatum seed oil. In a previous study, two genotypes of $A$. truncatum seeds were compared using a transcriptome analysis, and the candidate genes were revealed to affect seed VLCFA accumulation (Wang et al., 2018). During this study, we obtained comprehensive genomic data characterizing gene expressions at six time points of seed development in the $A$. truncatum tree. A total of 28,438 genes were identified. The oil component results showed that $85 \mathrm{DAF}$ was the key time point for FA synthesis during seed development. Therefore, we conducted a differential expression analysis by comparing other time points with $85 \mathrm{DAF}$. The results revealed that the numbers of upregulated and downregulated genes were similar at the different time points, except for 145 vs. 85 DAF. More importantly, some DEGs were associated with FA metabolism, including UFA biosynthesis, suberin and wax biosynthesis, FA biosynthesis, FA degradation, and FA elongation (34, 42, 44, 42, and 45 DEGs, respectively). In addition, we identified 20 DEGs associated with linoleic acid metabolism. The current study provides large-scale transcriptomics data for future research of lipid metabolism.

KEY GENES OF A. TRUNCATUM FATTY ACID BIOSYNTHESIS AND OIL ACCUMUlation. Oil synthesis mainly includes de novo FA biosynthesis, TAG assembly, and oil body formation in oil seed plants (Liu et al., 2013). In general, these genes are clearly part of the main pathways of FA and oil biosynthesis (Liao et al., 2018; Wan et al., 2016; Wang et al., 2019; Wu et al., 2020). However, the numbers and expression patterns of key genes vary from species to species during the process of seed development. In this study, we identified the key genes of FA biosynthesis and oil accumulation in A. truncatum seeds (Fig. 4). A. truncatum is rich in oleic acid (C18:1) and linoleic acid (C18:2), which account for $20.93 \%$ and $30.21 \%$ of its seed oil, respectively. Some studies have reported that C18:1-ACP is catalyzed by $S A D$ from C18:0-ACP (Liao et al., 2018; Liu et al., 2019; Wang et al., 2019). We identified three SADhomologous genes. Although SADl showed the highest FPKM values, its expression trend decreased from 85 DAF. Previous studies have reported that members of the FAD family have important roles in the biosynthesis of linoleic acid and linolenic acid (Alotaibi et al., 2020; Liao et al., 2018; Liu et al., 2019). In A. truncatum, six FAD genes were identified, which is less than that in A. thaliana (15), $S$. indicum (7), O. europaea (8), and G. $\max (15)$. Transgenic $A$. thaliana seeds overexpressing FAD2 and FAD3 from Paeonia suffruticosa contained more linolenic acid than wild-type seeds (Yin et al., 2018; Zhang et al., 2019). Based on the qRT-PCR and RNA-seq analyses, FAD2/6 were highly expressed and $F A D 3 / 7 / 8$ showed low expression. A previous study indicated that $F A D 3 / 7 / 8$ are $\omega-3$ desaturases that insert a double bond in linoleic acid (18:2) to synthesize linolenic acid (C18:3) (Wang et al., 2019). Furthermore, they are primarily responsible for the (18:3) content of seeds (Hernández et al., 2016). In $A$. truncatum, the highest content of C18:3 was only $0.92 \%$ at $145 \mathrm{DAF}$. Therefore, this may be the main reason for the high linoleic and low linolenic acid contents in A. truncatum. 
TAG is stored in oil bodies surrounded by a lipid monolayer and is associated with oil body membrane proteins (Frandsen et al., 2001). Fan et al. (2013) reported that overexpression of PDAT stimulated FA and TAG synthesis, and that coexpression of $P D A T$ with oleosin increased the dry weight of triacylglycerol by up to $6.4 \%$. Additionally, $D G A T$ works in coordination with $P D A T$ to enhance TAG assembly in seeds (Hong and Seo, 2018). Two PDAT genes and one $D G A T$ gene were identified in $A$. truncatum. These genes have the potential to increase the FA content and TAG synthesis in A. truncatum in the future.

Transcription FACTORS INVOLVED IN OIL ACCUMUlation. Regulation of FA biosynthesis has been shown to occur at multiple levels, among which transcriptional regulation is considered a major means of regulating the pathway (Millar et al., 2000). Previous studies showed that LEC1, WRI1, FUS3, and $A B I 3$ are key regulators of FA biosynthesis (Baud et al., 2007; Mu et al., 2008). For example, overexpression of maize (Zea mays) LEC1 (ZmLEC1) and WRI1 (ZmWRI1) increased oil accumulation by $\approx 48 \%$ in maize seeds (Shen et al., 2010). Transient expression of a Ricinus communis WRI1 (RcWRI1) splicing isoform in Nicotiana tabacum leaves upregulated the expression of WRI1 target genes and increased the oil content by 4.3-fold to 4.9-fold compared with controls (Ji et al., 2018). The $A B I 3$ could promote oleosin gene expression in B. napus (Crowe et al., 2000). In general, the regulatory mechanism of FA metabolism is complicated and may need several TFs to work together. To validate the expression differences in the identified TFs, we analyzed their expression trends at different seed development stages by using qRT-PCR. The results showed that the expression trends of WRII and LEC1.1 increased during seed development, but that FUS3 expression decreased from 85 to 180 DAF. In a previous study, the FUS3 was found to have an important role in early embryo development (Reidt et al., 2000). This may be the reason for its high expression during early seed development. These results provide some valuable clues to understanding the molecular mechanism of FA and lipid biosynthesis. However, further studies are needed to decipher the regulatory roles of TFs in $A$. truncatum, including identifying the binding motifs of the TFs that interact with the promoters of genes involved in oil biosynthesis.

\section{Conclusions}

In this study, oil accumulation during seed development showed an "S" pattern from 70 to 180 DAF; 85 DAF was the key time point for FA biosynthesis, when the main FA components were detected. To further understand the basic molecular mechanisms of lipid biosynthesis and metabolism, RNAseq data were generated from $A$. truncatum seeds at six developmental stages. GO and KEGG pathway enrichment analyses revealed that the DEGs were involved in biological processes such as FA and TAG biosynthesis. In addition, genes encoding key enzymes and TFs involved in the biosynthesis and metabolism of FAs and TAG were successfully identified. This is the first comprehensive transcriptomic analysis of the metabolic pathways of oleic acid, linoleic acid, linolenic acid, and TAG biosynthesis. The results will serve as an important foundation for more deeply exploring the regulatory mechanism of oil accumulation in this species and may provide a reference for other oil crops.

\section{Literature Cited}

Alotaibi, S.S, M.M. Elseehy, B.S. Aljuaid, and A.M. El-Shehawi. 2020. Transcriptome analysis of jojoba (Simmondsia chinensis) during seed development and liquid wax ester biosynthesis. Plants 9:588, doi: 10.3390/plants9050588.

Ashburner, M., C.A. Ball, J.A. Blake, D. Botstein, H. Butler, J.M. Cherry, A.P. Davis, K. Dolinski, S.S. Dwight, J.T. Eppig, M.A. Harris, D.P. Hill, L. Issel-Tarver, A. Kasarskis, S. Lewis, J.C. Matese, J.E. Richardson, M. Ringwald, G.M. Rubin, and G. Sherlock. 2000. Gene ontology: Tool for the unification of biology. Nat. Genet. 25:25-29, doi: 10.1038/75556.

Baud, S., M.S. Mendoza, A. To, E. Harscoët, L. Lepiniec, and B. Dubreucq. 2007. WRINKLED1 specifies the regulatory action of LEAFY COTYLEDON2 towards fatty acid metabolism during seed maturation in Arabidopsis. Plant J. 50:825-838, doi: 10.1111/j.1365313X.2007.03092.x.

Chen, D.J., L.H. Yan, Q. Li, C.J. Zhang, C.L. Si, Z.Y. Li, Y.J. Song, H. Zhou, T.C. Zhang, and X.G. Luo. 2017. Bioconversion of conjugated linoleic acid by Lactobacillus plantarum CGMCC8198 supplemented with Acer truncatum Bunge seeds oil. Food Sci. Biotechnol. 26:1595-1611, doi: 10.1007/s10068-017-0218-8.

Crowe, A.J., M. Abenes, A. Plant, and M. Maurice. 2000. The seedspecific transactivator, $\mathrm{ABI} 3$, induces oleosin gene expression. Plant Sci. 151:171-181, doi: 10.1016/S0168-9452(99)00214-9.

Dahlqvist, A., U. Stahl, M. Lenman, A. Banas, M. Lee, L. Sandager, H. Ronne, and S. Stymne. 2000. Phospholipid:diacylglycerol acyltransferase: An enzyme that catalyzes the acyl-CoA-independent formation of triacylglycerol in yeast and plants. Proc. Natl. Acad. Sci. USA 97:6487-6492, doi: 10.1073/pnas.120067297.

Dong, C.J., N. Cao, Z.G. Zhang, and Q.M. Shang. 2016. Characterization of the fatty acid desaturase genes in cucumber: structure, phylogeny, and expression patterns. PLoS ONE 11:e0149917, doi: 10.1371/journal.pone.0149917.

Fan, J., C. Yan, X. Zhang, and C. Xu. 2013. Dual role for phospholipid:diacylglycerol acyltransferase: Enhancing fatty acid synthesis and diverting fatty acids from membrane lipids to triacylglycerol in Arabidopsis leaves. Plant Cell 25:3506-3518, doi: 10.1105/ tpc.113.117358.

Frandsen, G.I., J. Mundy, and J.T.C. Tzen. 2001. Oil bodies and their associated proteins, oleosin and caleosin. Physiol. Plant. 112:301307, doi: 10.1034/j.1399-3054.2001.1120301.x.

Gao, J. 2006. Experimental guidance for plant physiology. China Higher Educ. Press, Beijing, China (in Chinese).

Grover, A., M. Kumari, S. Singh, S.S. Rathode, S.M. Gupta, P. Pandey, S. Gilotra, D. Kumar, M. Arif, and Z. Ahmed. 2014. Analysis of Jatropha curcas transcriptome for oil enhancement and genic markers. Physiol. Mol. Biol. Plants 20:139-142, doi: 10.1007/ s12298-013-0204-4.

Hernández, M.L., M.D. Sicardo, and J.M. Martínez-Rivas. 2016. Differential contribution of endoplasmic reticulum and chloroplast $\omega-3$ fatty acid desaturase genes to the linolenic acid content of olive (Olea europaea) fruit. Plant Cell Physiol. 57:138151, doi: $10.1093 / \mathrm{pcp} / \mathrm{pcv} 159$.

Hong, G.L. and P.J. Seo. 2018. Interaction of DGAT1 and PDAT1 to enhance tag assembly in Arabidopsis. Plant Signal. Behav. 14:1554467, doi: 10.1080/15592324.2018.1554467.

Hu, P., X. Xu, and L. Yu. 2017. Physicochemical properties of Acer truncatum seed oil extracted using supercritical carbon dioxide. J. Amer. Oil Chem. Soc. 94:779-786, doi: 10.1007/s11746-017-2983-1.

Ji, X.J., X. Mao, Q.T. Hao, B.L. Liu, J.A. Xue, and R.Z. Li. 2018. Splice variants of the castor WRI1 gene upregulate fatty acid and oil biosynthesis when expressed in tobacco leaves. Intl. J. Mol. Sci. 19:146, doi: 10.3390/ijms19010146.

Jiao, G., J.P. Hui, I.W. Burton, M.H. Thibault, C. Pelletier, J. Boudreau, N. Tchoukanova, B. Subramanian, Y. Djaoued, S. Ewart, J. Gagnon, K.V. Ewart, and J. Zhang. 2015. Characterization of shrimp oil from Pandalus borealis by high performance liquid 
chromatography and high resolution mass spectrometry. Mar. Drugs 13:3849-3876, doi: 10.3390/md13063849.

Jones, A., H.M. Davies, and T.A. Voelker. 1995. Palmitoyl-acyl carrier protein (ACP) thioesterase and the evolutionary origin of plant acylACP thioesterases. Plant Cell 7:359-371, doi: 10.1105/tpc.7.3.359.

Kanehisa, M., M. Araki, S. Goto, M. Hattori, M. Hirakawa, M. Itoh, T. Katayama, S. Kawashima, S. Okuda, T. Tokimatsu, and Y. Yamanishi. 2008. KEGG for linking genomes to life and the environment. Nucleic Acids Res. 36:480-484, doi: 10.1093/nar/gkm882.

Kim, D., B. Langmead, and S.L. Salzberg. 2015. HISAT: A fast spliced aligner with low memory requirements. Nat. Methods 12:357-360, doi: $10.1038 /$ nmeth.3317.

Kirk, R.E. 2006. Fisher, Ronald Alymer, p. 354. In: N.J. Salkind (ed.). Encyclopedia of measurement and statistics. Sage, Thousand Oaks, CA.

Liang, Q., W.W. Wang, F.L. Yuan, X. Liu, D.L. Li, and K.Q. Yang. 2019. Characterization of yuanbaofeng (Acer truncatum Bunge) samaras: Oil, fatty acid, and phytosterol content. Ind. Crops Prod. 135:344-351, doi: 10.1016/j.indcrop.2019.04.032.

Liao, B., Y.J. Hao, J.X. Lu, H.Y. Bai, L. Guan, and T. Zhang. 2018. Transcriptomic analysis of Perilla frutescens seed to insight into the biosynthesis and metabolic of unsaturated fatty acids. BMCC Genomics 19:213, doi: 10.1186/s12864-018-4595-z.

Liu, H., J. Gu, Q. Lu, H.F. Li, Y.B. Hong, X.P. Chen, L. Ren, L. Deng, and X.Q. Liang. 2019. Transcriptomic analysis reveals the high-oleic acid feedback regulating the homologous gene expression of stearoyl-ACP desaturase $2(S A D 2)$ in peanuts. Intl. J. Mol. Sci. 20:3091, doi: 10.3390/ijms20123091.

Liu, X.Y., H. Fu, and Y.H. Chen. 2003. Study on the physico-chemical properties and fatty acid composition of Acer truncatum Buge oil. China Oils Fats 28:66-67 (in Chinese), doi: 10.3321/j.issn:10037969.2003.03.021.

Liu, Y.L., Z.D. Huang, Y. Ao, W. Li, and Z.X. Zhang. 2013. Transcriptome analysis of yellow horn (Xanthoceras sorbifolia Bunge): A potential oil-rich seed tree for biodiesel in china. PLoS One 9:e 74441, doi: 10.1371/annotation/803f7e8c-0718-41b4-8fc2cc0b5f776da9.

Livak, K.J. and T.D. Schmittgen. 2001. Analysis of relative gene expression data using real-time quantitative PCR and the $2^{-\Delta \Delta C T}$ method. Methods 25:402-408, doi: 10.1006/meth.2001.

Lung, S.C. and R.J. Weselake. 2006. Diacylglycerol acyltransferase: A key mediator of plant triacylglycerol synthesis. Lipids 41:10731088, doi: 10.1007/s11745-006-5057-y.

Ma, Q.Y., T.L. Sun, S.S. Li, J. Wen, Lu. Zhu, T.M. Yin, K.Y. Yan, X. Xu, S.X. Li, J.F. Mao, Y.N. Wang, S.X. Jin, X. Zhao, and Q.Z. Li. 2020. The Acer truncatum genome provides insights into the nervonic acid biosynthesis. Plant J., doi: 10.1111/tpj.14954.

Ma, X.F., W.X. Tian, L.H. Wu, X.L. Cao, and Y. Ito. 2005. Isolation of quercetin-3-O-L-rhamnoside from Acer truncatum Bunge by highspeed counter-current chromatography. J. Chromatography 1070:211-214, doi: 10.1016/j.chroma.2005.02.052.

Mao, X.Z., T. Cai, J.G. Olyarchuk, and L. Wei. 2015. Automated genome annotation and pathway identification using the KEGG orthology (KO) as a controlled vocabulary. Bioinformatics 21:37873793, doi: $10.2307 / 1592215$.

Millar, A.A., M.A. Smith, and L. Kunst. 2000. All fatty acids are not equal: Discrimination in plant membrane lipids. Trends Plant Sci. 5:95-101, doi: 10.1016/S1360-1385(00)01566-1.

Mu, J.Y., H.L. Tan, Q. Zheng, F.Y. Fu, Y. Liang, J. Zhang, X.H. Yang, T. Wang, K. Chong, X.J. Wang, and J.R. Zuo. 2008. LEAFY COTYLEDON1 is a key regulator of fatty acid biosynthesis in Arabidopsis. Plant Physiol. 48:1042-1054, doi: 10.1104/ pp.108.126342.

Mudalkar, S., R. Golla, S. Ghatty, and A.R. Reddy. 2014. De novo transcriptome analysis of an imminent biofuel crop, Camelina sativa L. using Illumina GAIIX sequencing platform and identification of SSR markers. Plant Mol. Biol. 84:159-171, doi: 10.1007/s11103013-0125-1.
Muñoz-Mérida, A., J.J. González-Plaza, A. Cañada, A.M. Blanco, M. García-López, J.M. Rodríguez, L. Pedrola, M.D. Sicardo, M.L. Hernández, R. De la Rosa, A. Belaj, M. Gil-Borja, F. Luque, J.M. Martínez-Rivas, D.G. Pisano, O. Trelles, V. Valpuesta, and C.R. Beuzón. 2013. De novo assembly and functional annotation of the olive (Olea europaea) transcriptome. DNA Res. 20:93-108, doi: 10.1093/dnares/dss036.

Rawsthorne, S. 2002. Carbon flux and fatty acid synthesis in plants. Prog. Lipid Res. 41:182-196, doi: 10.1016/S0163-7827(01)00023-6.

Reidt, W., T. Wohlfarth, M. Ellerström, A. Czihal, A. Tewes, I. Ezcurra, L. Rask, and H. Bäumlein. 2000. Gene regulation during late embryogenesis: The RY motif of maturation-specific gene promoters is a direct target of the FUS3 gene product. Plant J. 21:401-408, doi: 10.1046/j.1365-313x.2000.00686.x.

Riechmann, J.L., J. Heard, G. Martin, L. Reuber, C.Z. Jiang, J. Keddie, L. Adam, O. Pineda, O.J. Ratcliffe, and R.R. Samaha. 2000. Arabidopsis transcription factors: Genomewide comparative analysis among eukaryotes. Science 290:2105, doi: 10.1126/science. 290.5499.2105.

Rohde, A., M.V. Montagu, and W. Boerjan. 2010. The ABSCISIC ACID-INSENSITIVE 3 ( $A B I 3$ ) gene is expressed during vegetative quiescence processes in Arabidopsis. Plant Cell Environ. 22:261270, doi:10.1046/j.1365-3040.1999.00428.x.

Shen, B., W.B. Allen, P. Zheng, C. Li, K. Glassman, J. Ranch, D. Nubel, and M.C. Tarczynski. 2010. Expression of ZmLEC1 and ZmWRII increases seed oil production in maize. Plant Physiol. 153:980-987, doi: 10.1104/pp.110.157537.

Tamura, K., D. Peterson, N. Peterson, G. Stecher, M. Nei, and S. Kumar. 2011. MEGA5: Molecular evolutionary genetics analysis using maximum likelihood, evolutionary distance, and maximum parsimony methods. Mol. Biol. Evol. 28:2731-2739, doi: 10.1093/ molbev/msr121.

Wan, H.F., Y.X. Cui, T.J. Ding, J.Q. Mei, H.L. Dong, W.X. Zhang, S.Q. Wu, Y. Liang, C.Y. Zhang, J.N. Li, Q. Xiong, and W. Qian. 2016. Time-series analyses of transcriptomes and proteomes reveal molecular networks underlying oil accumulation in canola. Front. Plant Sci 7:2007, doi: 10.3389/fpls.2016.02007.

Wang, L., Z. Feng, X. Wang, X.W. Wang, and X.G. Zhang. 2010. DEGseq: An R package for identifying differentially expressed genes from RNA-seq data. Bioinformatics 26:136-138, doi: 10.1093/ bioinformatics/btp612.

Wang, L., W. Shen, M. Kazachkov, G. Chen, Q. Chen, A.S. Carlsson, S. Stymne, R.J. Weselake, and J. Zou. 2012. Metabolic interactions between the lands cycle and the Kennedy pathway of glycerolipid synthesis in Arabidopsis developing seeds. Plant Cell 24:4652-4669, doi: $10.1105 /$ tpc.112.104604.

Wang, R.K., P. Liu, J.S. Fan, and L.L. Li. 2018. Comparative transcriptome analysis two genotypes of Acer truncatum Bunge seeds reveals candidate genes that influences seed VLCFAs accumulation. Sci. Rep. 8:15504, doi: 10.1038/s41598-018-33999-3.

Wang, X.J., H.Y. Liang, D.L. Guo, X.G. Duan, Q.S. Jia, and X.G. Hou. 2019. Integrated analysis of transcriptomic and proteomic data from tree peony $(P$. ostii) seeds reveals key developmental stages and candidate genes related to oil biosynthesis and fatty acid metabolism. Hort. Res. 6:111, doi: 10.1038/s41438-019-0194-7.

Wang, X.Y., J.S. Fan, S.Y. Wang, and R.C. Sun. 2006. A new resource of nervonic acid from purpleblow maple (Acer truncatum) seed oil. For. Prod. J. 56:147-150, doi: 10.1007/s00226-006-0085-6.

Wu, Q.K., Y. Cao, C. Chen, Z. Gao, and R.D. Guy. 2020. Transcriptome analysis of metabolic pathways associated with oil accumulation in developing seed kernels of styrax tonkinensis, a woody biodiesel species. BMC Plant Biol. 20: doi: 10.1186/s12870-0202327-4.

Yang, L.G., P.P. Yin, H. Fan, Q. Xue, K. Li, X. Li, L.W. Sun, and Y.J. Liu. 2017. Response surface methodology optimization of ultrasonic-assisted extraction of Acer truncatum leaves for maximal phenolic yield and antioxidant activity. Molecules 22:232, doi: $10.3390 /$ molecules 22020232 . 
Yin, D.D., X.W. Zhong, Q.Y. Shu, S.S. Li, Q. Wu, C.Y. Feng, Z.Y. Gu, and L.S. Wang. 2018. Fatty acid desaturase 3 (PsFAD3) from Paeonia suffruticosa reveals high $\alpha$-linolenic acid accumulation. Plant Sci. 274:212-222, doi: 10.1016/j.plantsci.2018.05.027.

Zhang, Z., Y. Luo, X. Wang, and F. Yu. 2018. Fruit spray of 24epibrassinolide and fruit shade alter pericarp photosynthesis activity and seed lipid accumulation in Styrax tonkinensis. J. Plant Growth Regul. 37:1066-1084, doi: 10.1007/s00344-017-9769-4.

Zhang, Q.Y., R. Yu, D.Y. Sun, M. Raham, L.H. Xie, J.Y. Hu, L.X. He, A. Kilaru, L.X. Niu, and Y.L. Zhang. 2019. Comparative transcriptome analysis reveals an efficient mechanism for $\alpha$-linolenic acid synthesis in tree peony seeds. Intl. J. Mol. Sci. 20:65, doi: 10.3390/ijms20010065.
Zhang, M., X. Cao, Q. Jia, Q.L. Jia, and J. Ohlrogge. 2016. FUSCA3 activates triacylglycerol accumulation in Arabidopsis seedlings and tobacco BY2 cells. Plant J. 88:95-107, doi: 10.1111/tpj.13233.

Zhao, W.H., J.F. Zhang, Z. Wang, Y.X. Zhang, and W.X. Tian. 2006. The extract of leaves of Acer truncatum Bunge: A natural inhibitor of fatty acid synthase with antitumor activity. J. Enzyme Inhib. Med. Chem. 21:589-596, doi: 10.1080/14756360600774579.

Zheng, Y., C. Jiao, H. Sun, H.G. Rosli, M.A. Pombo, P. Zhang, M. Banf, X. Dai, G.B. Martin, J.J. Giovannoni, P.X. Zhao, S.Y. Rhee, and Z.J. Fei. 2016. iTAK: A program for genome-wide prediction and classification of plant transcription factors, transcriptional regulators, and protein kinases. Mol. Plant 9:1667-1670, doi: 10.1016/ j.molp.2016.09.014. 
A

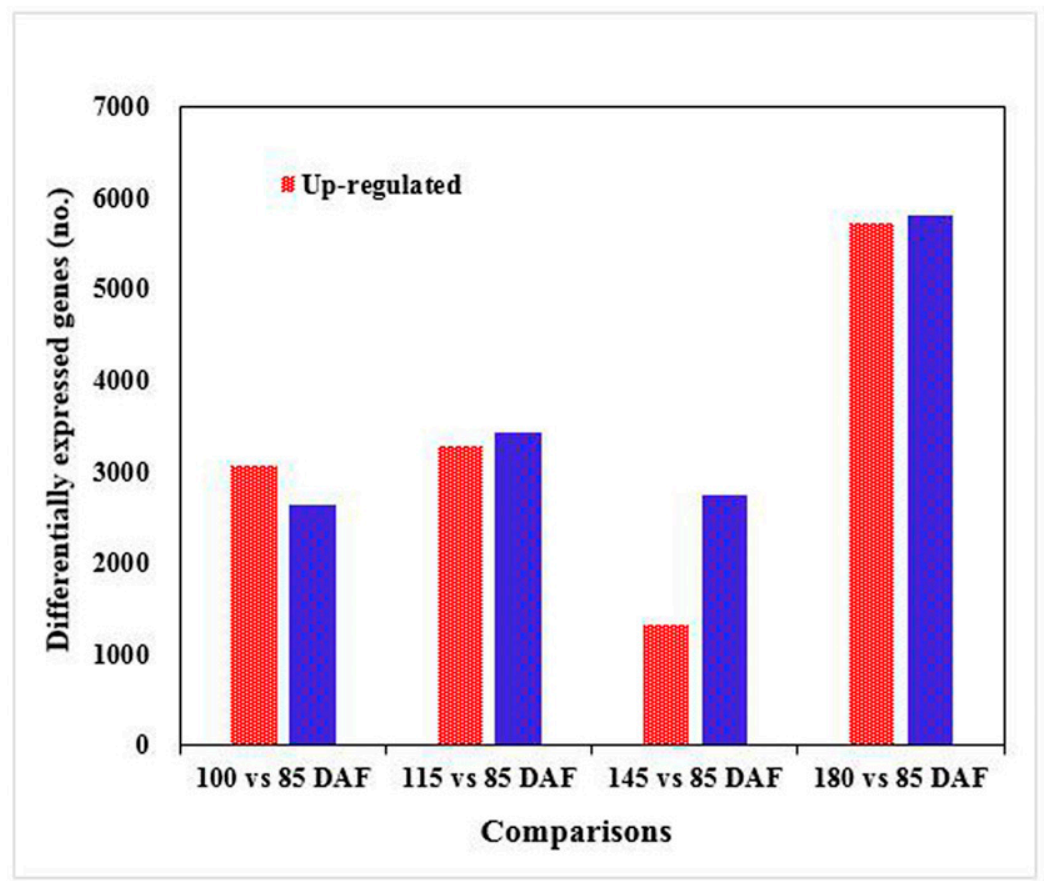

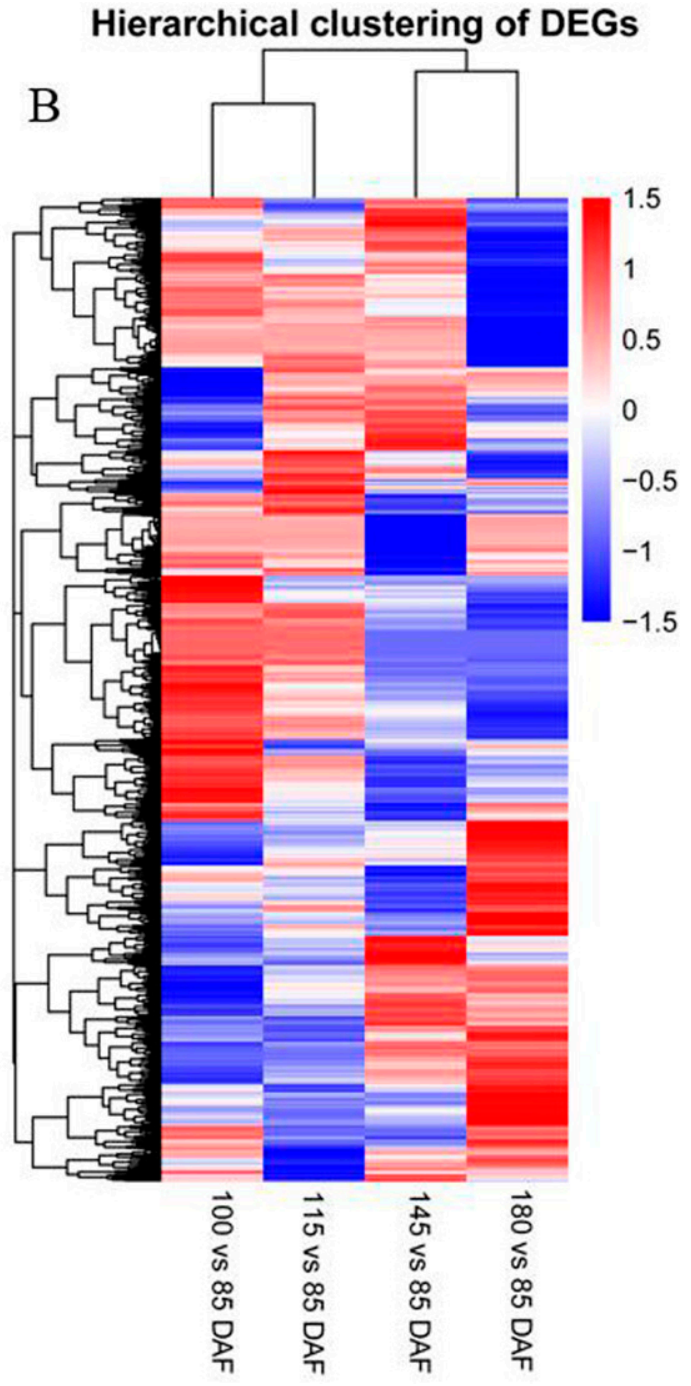

Supplemental Fig. 1. Differentially expressed genes (DEGs) identified in Acer truncatum seeds at various development stages: (A) quantity of DEGs and (B) cluster analysis of DEGs. The heat map is displayed according to $\log _{10}$ [fragments per kilobase per million $(\mathrm{FPKM})+1$ ] values. DAF $=$ days after flowering. 

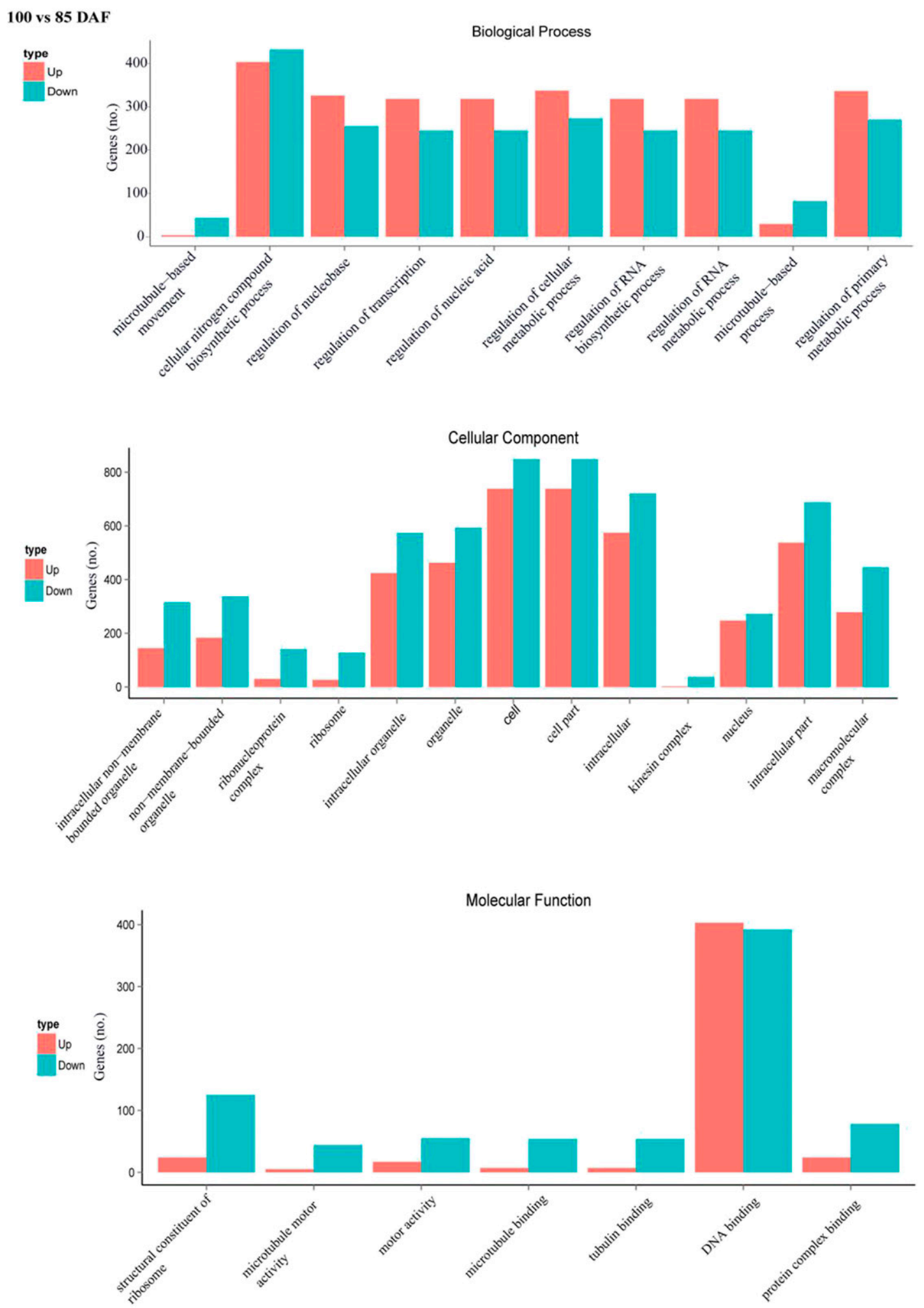

Supplemental Fig. 2. Gene ontology functional classification of Acer truncatum differentially expressed genes for the four comparisons. $\mathrm{DAF}=\mathrm{days}$ after flowering. 

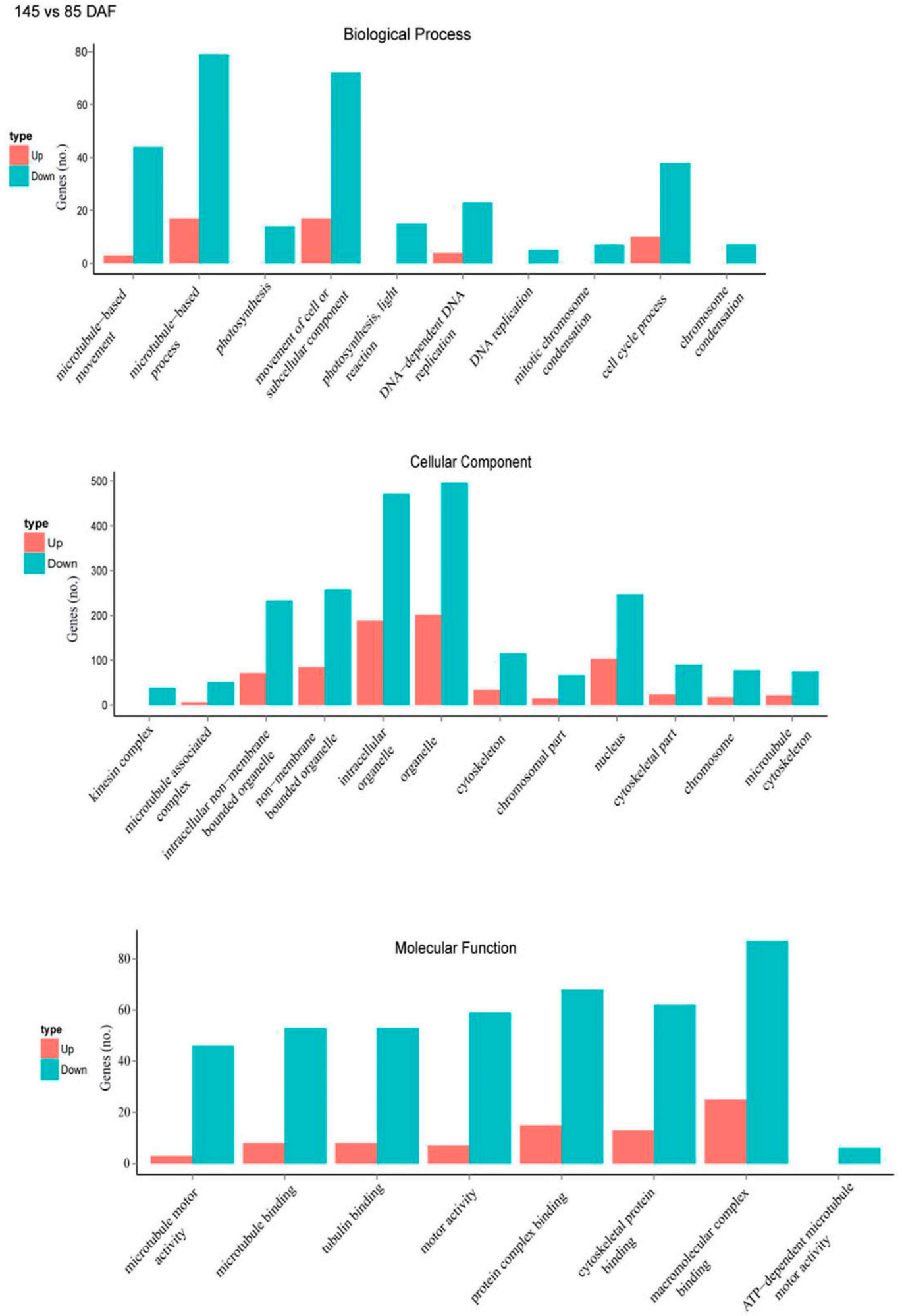

Supplemental Fig. 2. Cont. 
180 vs 85 DAF
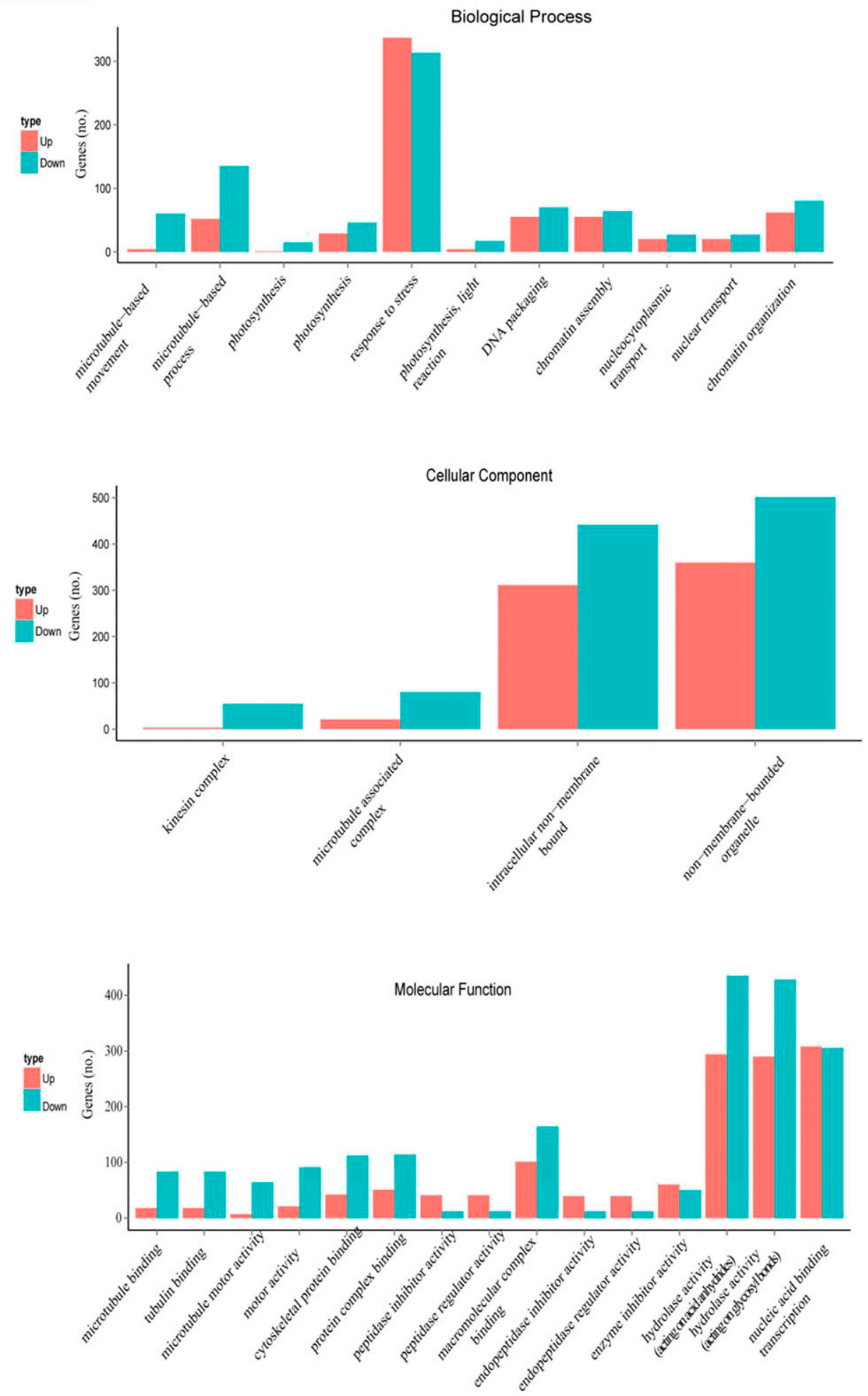

Supplemental Fig. 2. Cont. 

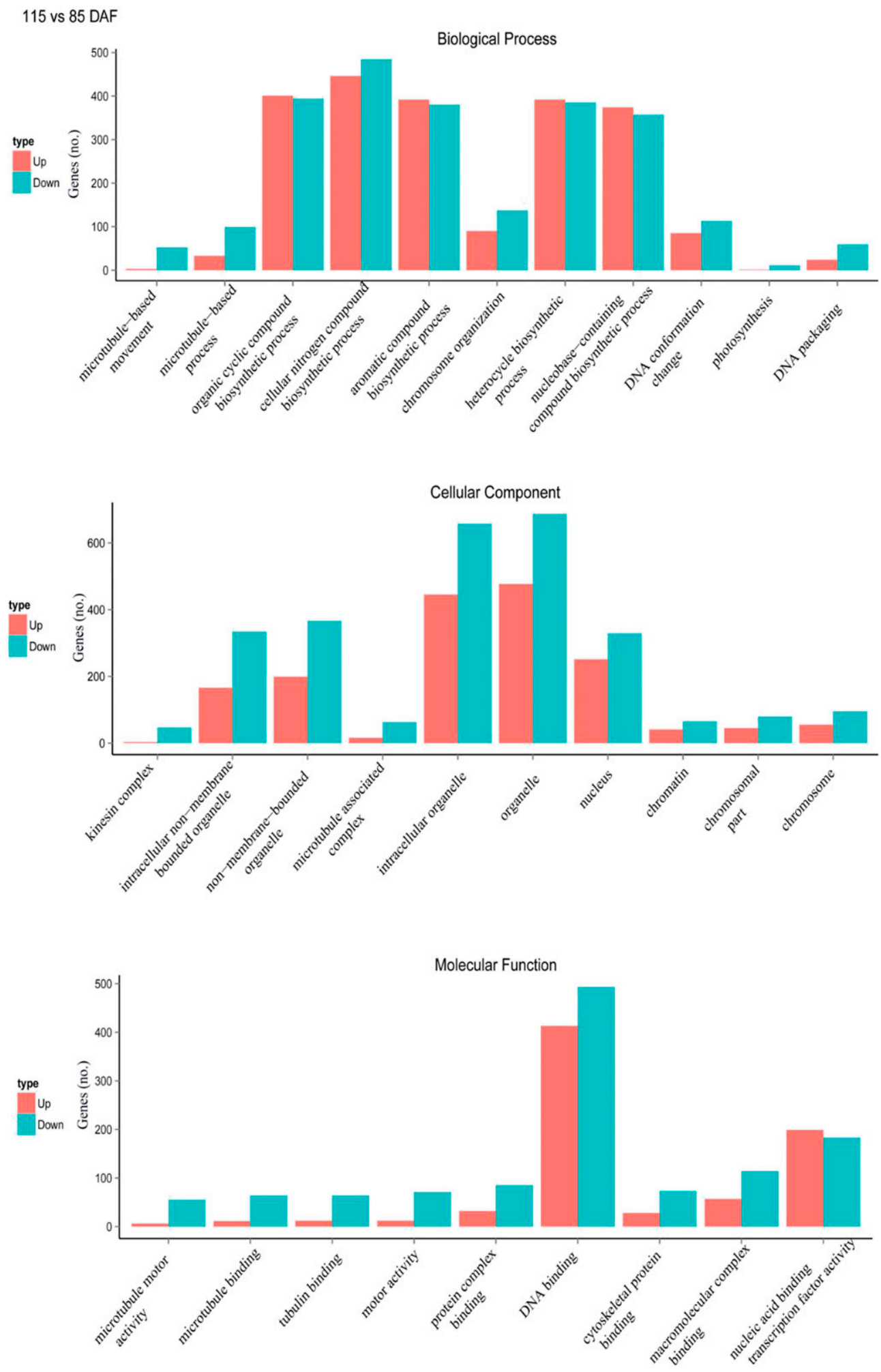

Supplemental Fig. 2. Cont. 
KEGG Classification

Environmental adaptation

Xenobiotics biodegradation and metabolism

Overview

Nucleotide metabolism

Metabolism of terpenoids and polyketides

Metabolism of other amino acids

Metabolism of cofactors and vitamins

Lipid metabolism

Glycan biosynthesis and metabolism

Energy metabolism

Carbohydrate metabolism

Biosynthesis of other secondary metabolites

Amino acid metabolism

Translation

Transcription

Replication and repair

Folding, sorting and degradation
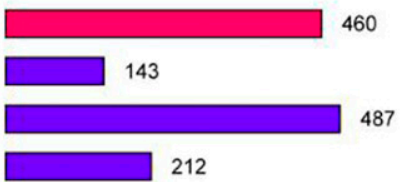

460
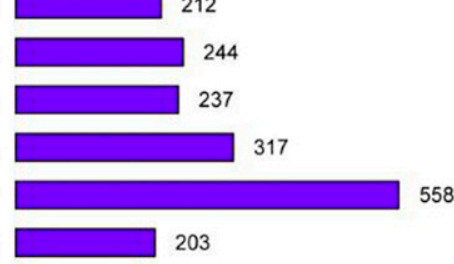

387

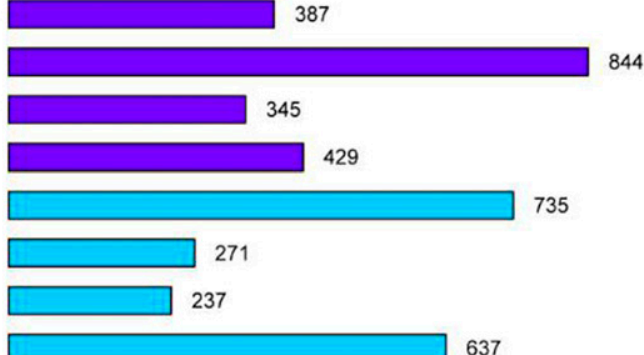

844

Signaling molecules and interaction

Signal transduction

Membrane transport

Transport and catabolism

10

Cellular commiunity 169

Cell motility

Cell growth and death

73

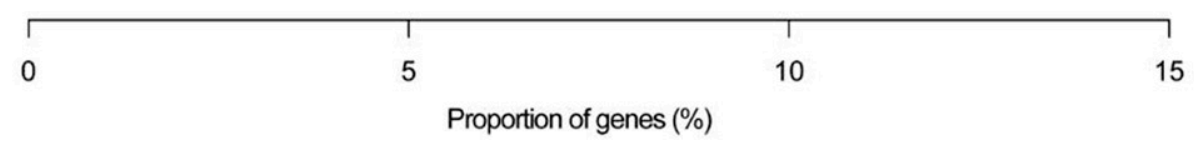

Supplemental Fig. 3. Histogram of clusters of Kyoto Encyclopedia of Genes and Genomes (KEGG) pathways for the differentially expressed genes in different Acer truncatum seed developmental stages. The horizontal axis represents the percentage of genes and the vertical axis shows the names of the KEGG clusters: (A) cellular processes; (B) environmental information processing; (C) genetic information processing; (D) metabolism; and (E) organismal systems. 

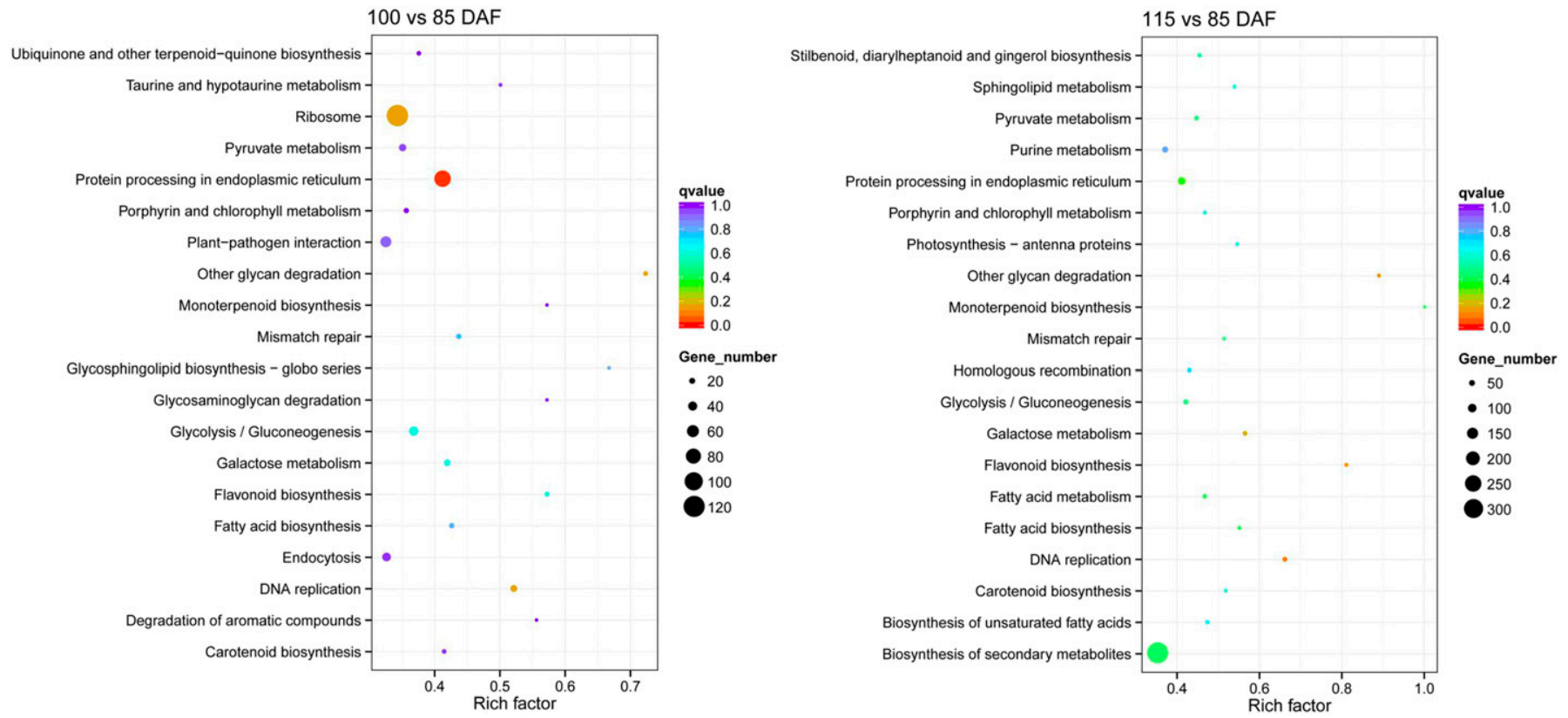

Supplemental Fig. 4. Results of the Kyoto Encyclopedia of Genes and Genomes (KEGG) enrichment analysis of Acer truncatum differentially expressed genes for the four comparisons. DAF = days after flowering.
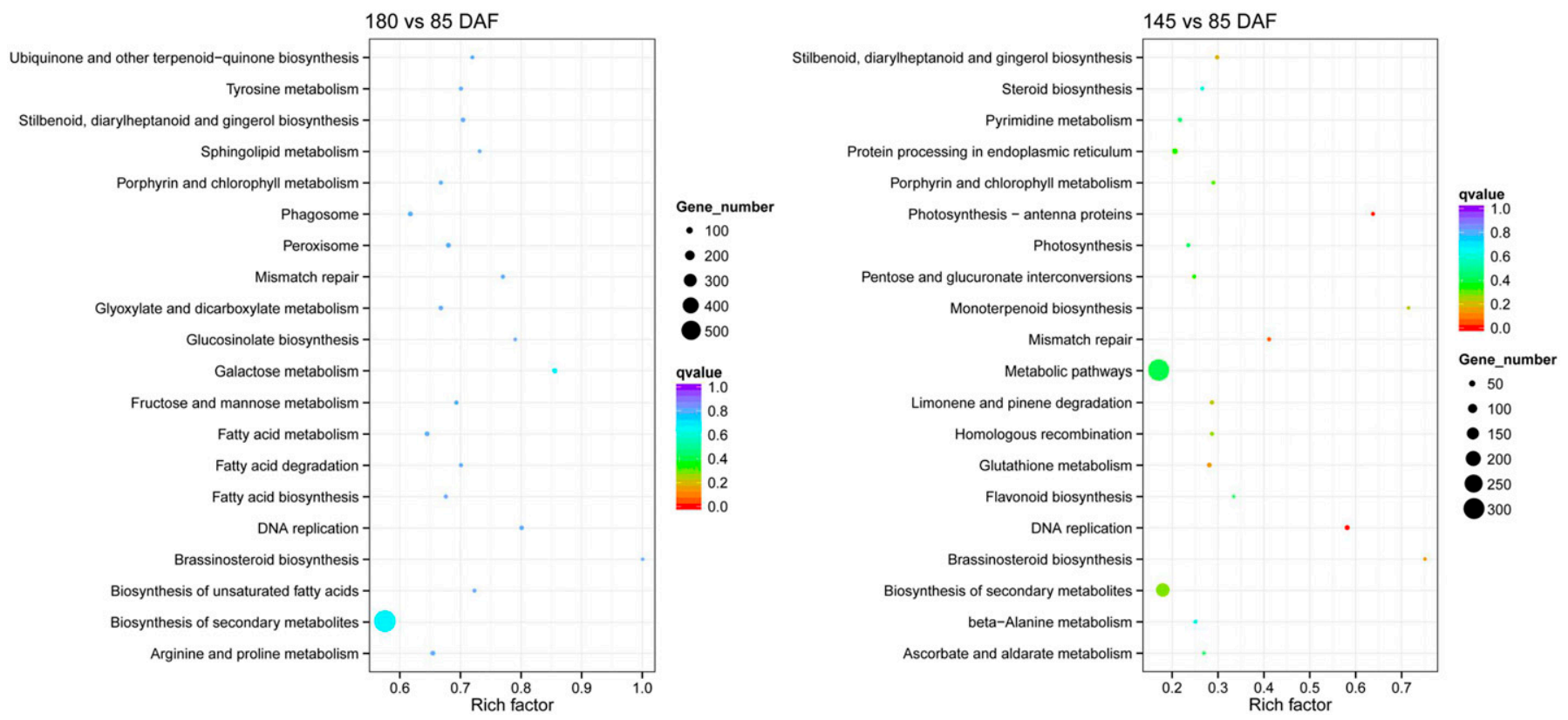

Supplemental Fig. 4. Cont. 
Supplemental Table 1. Primers $\left(5^{\prime}-3^{\prime}\right)$ used in the quantitative real-time polymerase chain reaction (qRT-PCR) to validate the reliability of the RNA-seq results.

\begin{tabular}{|c|c|c|}
\hline Gene & Forward primer & Reverse primer \\
\hline$\overline{\mathrm{ACC}}$ & TGAAGGTATTTCCGCTATTT & TCCACTTGCCACTACTATCC \\
\hline FATA1 & TCCTGCTTGGAGTGATGTGG & TTTCTGAAGTCGCCTGGTGT \\
\hline FATA2 & TCCTGCTTGGAGTGATGT & GTCGCCTGGTGTCTTGAT \\
\hline FATB & GAACTCTGACCCTATTGTGG & ATCCAGCCAATGTATTTCAC \\
\hline SAD1 & ACCACCATTCTCCACCGTCAG & TTGCCAGCATTTATCTACCG \\
\hline SAD2 & CAATGGCCTCCACTCTTCGT & TTATCCTCAGCCCAACCCTC \\
\hline SAD3 & GGCCTCCACTCTTCGCTCCA & CCTCCGCCCAATCCTCCATA \\
\hline FAD2 & TGGTCCCTTATTTCTCGTGG & AATTTCTGCCTTGGTCTTGG \\
\hline FAD2 & TGACCCTTACGATTACACTTAC & AATAACGGCACTCCATACAC \\
\hline FAD3 & CCGAGGAAAGGAATGGAGTT & GTGAGGGATTTGAGGGAAGA \\
\hline FAD5 & TACCACTCCACCGACCAAAC & TATTCGCTTCTGCTTCAACT \\
\hline FAD6 & ATGGTTGGGCTATCACTTCT & CTCAATCCAACGAGGGTAAT \\
\hline FAD $7 / 8$ & CCCGAGTGAGATGAAAGATG & CAAGTAAGTCACGAAATCCAG \\
\hline PDAT1 & CAGTTGCTTCTACTAGGTGGT & AACAGGTGTCCACGCACGAC \\
\hline PDAT2 & CGGCGACGACATACAGGAAA & AGCAACCACCATATCGAGCA \\
\hline DGAT & AAGAGGATTTGTTCGCATAG & GCATTGGTTGTTTATAGGGT \\
\hline $\mathrm{ABI} 3$ & GAAGTCCGATACAGATGAAGG & GATGCCGTAGAAGACAAAGC \\
\hline LEC1 & TTGATGACTACATTGAGCCGTTGA & GCAGCAGGTGCGAAAGAAGC \\
\hline LEC1.1 & CTCACTTGGAGCACAACTAAC & CTGCCATTACCATTCTCATC \\
\hline FUS3 & GACCAAACGGCTACTAATACG & GACTGGTAACAGCAGCGACA \\
\hline WRI1 & AGGCGAAACAAGGAGATAGT & TTGGGATGATTGATAGAGGG \\
\hline Actin & ATCATGTTTGAGACCTTCAACAC & GATCTTCATGAGGTAGTCAGTCAGGT \\
\hline
\end{tabular}

Supplemental Table 2. Summary of sequencing data of Acer truncatum seeds and alignment information of clean reads.

\begin{tabular}{|c|c|c|c|c|c|c|c|}
\hline Sample $^{z}$ & Raw reads & Clean reads & $\begin{array}{l}\text { Clean } \\
\text { bases }\end{array}$ & $\begin{array}{c}\text { Clean data } \\
\text { rate }(\%)\end{array}$ & $\begin{array}{l}\text { Total mapped } \\
\text { reads }(\%)\end{array}$ & $\begin{array}{c}\text { Unique } \\
\text { matches }(\%)\end{array}$ & $\begin{array}{c}\text { Multi-position } \\
\text { matches (\%) }\end{array}$ \\
\hline 70DAF_1 & $51,614,678$ & $50,548,700$ & $7.58 \mathrm{G}$ & $97.93 \%$ & 90.32 & 83.52 & 6.80 \\
\hline 70DAF_2 & $37,144,834$ & $36,363,230$ & $5.45 \mathrm{G}$ & $97.90 \%$ & 89.71 & 81.79 & 7.93 \\
\hline 70DAF_3 & $46,343,392$ & $45,391,246$ & $6.81 \mathrm{G}$ & $97.95 \%$ & 89.45 & 81.52 & 7.93 \\
\hline 85DAF_1 & $56,718,166$ & $54,937,224$ & $8.24 \mathrm{G}$ & $96.86 \%$ & 91.01 & 83.84 & 7.17 \\
\hline 85DAF_2 & $49,957,250$ & $48,619,534$ & $7.29 \mathrm{G}$ & $97.32 \%$ & 91.16 & 84.08 & 7.07 \\
\hline 85DAF_3 & $57,092,212$ & $55,301,938$ & $8.3 \mathrm{G}$ & $96.86 \%$ & 90.92 & 83.46 & 7.46 \\
\hline 100DAF_1 & $49,137,600$ & $47,956,846$ & $7.19 \mathrm{G}$ & $97.60 \%$ & 90.62 & 83.31 & 7.31 \\
\hline 100DAF_2 & $60,755,562$ & $59,280,166$ & $8.89 \mathrm{G}$ & $97.57 \%$ & 90.61 & 83.23 & 7.38 \\
\hline 100DAF_3 & $51,269,214$ & $49,835,732$ & $7.48 \mathrm{G}$ & $97.20 \%$ & 90.88 & 83.05 & 7.82 \\
\hline 115DAF_1 & $46,211,774$ & $45,051,936$ & $6.76 \mathrm{G}$ & $97.49 \%$ & 89.84 & 82.44 & 7.40 \\
\hline 115DAF_2 & $46,399,842$ & $45,279,334$ & $6.79 \mathrm{G}$ & $97.59 \%$ & 90.36 & 82.90 & 7.46 \\
\hline 115DAF_3 & $49,317,264$ & $48,307,076$ & $7.25 \mathrm{G}$ & $97.95 \%$ & 91.24 & 83.86 & 7.39 \\
\hline 145DAF_1 & $40,162,964$ & $38,493,262$ & $5.77 \mathrm{G}$ & $95.84 \%$ & 90.20 & 77.37 & 12.83 \\
\hline 145DAF_2 & $50,623,568$ & $48,784,840$ & $7.32 \mathrm{G}$ & $96.37 \%$ & 91.35 & 78.85 & 12.50 \\
\hline 145DAF_3 & $49,329,602$ & $47,496,066$ & $7.12 \mathrm{G}$ & $96.28 \%$ & 85.36 & 73.71 & 11.65 \\
\hline 180DAF_1 & $54,005,776$ & $52,803,466$ & $7.92 \mathrm{G}$ & $97.77 \%$ & 90.89 & 82.14 & 8.75 \\
\hline 180DAF_2 & $42,914,112$ & $41,795,622$ & $6.27 \mathrm{G}$ & $97.39 \%$ & 91.57 & 83.09 & 8.48 \\
\hline 180DAF_3 & $49,034,272$ & $47,528,266$ & $7.13 \mathrm{G}$ & $96.93 \%$ & 91.45 & 82.39 & 9.06 \\
\hline
\end{tabular}

${ }^{\mathrm{z}} \mathrm{DAF}=$ days after flowering. 
Supplemental Table 3. Significantly enriched Kyoto Encyclopedia of Genes and Genomes (KEGG) pathways of differentially expressed genes in Acer truncatum seeds from different developmental periods.

\begin{tabular}{|c|c|c|}
\hline Pathway & Genes (no.) & $P$ \\
\hline \multicolumn{3}{|l|}{100 vs. $85 \mathrm{DAF}^{\mathrm{z}}$} \\
\hline Ribosome & 122 & 0.004531798 \\
\hline Plant hormone signal transduction & 74 & 0.333131831 \\
\hline Carbon metabolism & 65 & 0.401327625 \\
\hline Flavonoid biosynthesis & 12 & 0.024871355 \\
\hline Fatty acid elongation & 7 & 0.682132256 \\
\hline Ether lipid metabolism & 7 & 0.522889655 \\
\hline Sesquiterpenoid and triterpenoid biosynthesis & 1 & 0.995839534 \\
\hline \multicolumn{3}{|l|}{115 vs. 85 DAF } \\
\hline Biosynthesis of secondary metabolites & 345 & 0.025954254 \\
\hline Biosynthesis of amino acids & 79 & 0.50474039 \\
\hline Flavonoid biosynthesis & 17 & 0.003249311 \\
\hline Starch and sucrose metabolism & 57 & 0.568025609 \\
\hline Fatty acid biosynthesis & 22 & 0.022698982 \\
\hline Unsaturated fatty acid biosynthesis & 17 & 0.099071687 \\
\hline Alpha-linolenic acid metabolism & 15 & 0.139017983 \\
\hline Fatty acid elongation & 12 & 0.302619961 \\
\hline Linoleic acid metabolism & 4 & 0.367364226 \\
\hline \multicolumn{3}{|l|}{145 vs. 85 DAF } \\
\hline Protein processing in endoplasmic reticulum & 45 & 0.036950766 \\
\hline Plant hormone signal transduction & 48 & 0.160450493 \\
\hline \multicolumn{3}{|l|}{180 vs. $85 \mathrm{DAF}$} \\
\hline Biosynthesis of secondary metabolites & 568 & 0.011740226 \\
\hline Biosynthesis of amino acids & 132 & 0.455449777 \\
\hline Plant hormone signal transduction & 130 & 0.729465793 \\
\hline Carbon metabolism & 125 & 0.480568449 \\
\hline Protein processing in endoplasmic reticulum & 111 & 0.550702943 \\
\hline Ribosome & 99 & 0.999999998 \\
\hline Unsaturated fatty acid biosynthesis & 26 & 0.109515596 \\
\hline Fatty acid degradation & 28 & 0.121109584 \\
\hline Fatty acid elongation & 18 & 0.37856479 \\
\hline Ether lipid metabolism & 12 & 0.668666136 \\
\hline
\end{tabular}

${ }^{\mathrm{z}} \mathrm{DAF}=$ days after flowering. 
Supplemental Table 4. All genes associated with lipid metabolism identified in the transcriptome.

\begin{tabular}{lcr}
\hline Lipid metabolism pathway & Genes with pathway annotation (no.) & Pathway \\
\hline Arachidonic acid metabolism & 34 & ko00590 \\
Biosynthesis of unsaturated fatty acids & 34 & ko01040 \\
Cutin, suberine, and wax biosynthesis & 42 & ko00073 \\
Ether lipid metabolism & 34 & ko00565 \\
Fatty acid biosynthesis & 44 & ko00061 \\
Fatty acid degradation & 42 & ko00071 \\
Fatty acid elongation & 45 & ko00062 \\
Glycerolipid metabolism & 117 & ko00561 \\
Glycerophospholipid metabolism & 116 & ko00564 \\
Linoleic acid metabolism & 20 & ko00591 \\
Primary bile acid biosynthesis & 1 & ko00120 \\
Sphingolipid metabolism & 80 & ko00600 \\
Steroid biosynthesis & 37 & ko00100 \\
Steroid hormone biosynthesis & 46 & ko00140 \\
Synthesis and degradation of ketone bodies & 6 & ko00072 \\
Alpha-linolenic acid metabolism & 56 & \\
\hline
\end{tabular}

